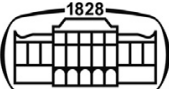

AKADÉMIAI KIADÓ

Journal of Behavioral Addictions

9 (2020) 1, 14-43

DOI:

$10.1556 / 2006.2020 .00005$

(c) 2020 The Author(s)

\section{REVIEW ARTICLE}

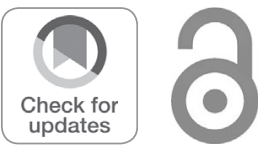

\title{
Treatments for internet addiction, sex addiction and compulsive buying: A meta-analysis
}

\author{
MARTINA GOSLAR $^{1 *} \odot$, MAX LEIBETSEDER ${ }^{1}$, HANNAH M. \\ MUENCH $^{2}$, STEFAN G. HOFMANN ${ }^{3}$ and ANTON-RUPERT \\ LAIREITER $^{1,4}$
}

\author{
${ }^{1}$ Department of Psychology, University of Salzburg, Hellbrunnerstrasse 34, 5020, Salzburg, Austria \\ ${ }^{2}$ Psychosomatisches Zentrum Waldviertel (PSZW), Universitätsklinik für Psychosomatische Medizin \\ der Karl Landsteiner Privat-Universität, Grafenbergerstraße 2, 3730, Eggenburg, Austria \\ ${ }^{3}$ Department of Psychological and Brain Sciences, Boston University, 900 Commonwealth Avenue, \\ 2nd Fl., Boston, MA, 02215, USA \\ ${ }^{4}$ Faculty of Psychology, University of Vienna, Liebiggasse 5, 1010, Wien, Austria
}

Received: July 26, 2019 • Revised manuscript received: December 28, 2019 • Accepted: January 25, 2020

Published online: April 7, 2020
*Corresponding author. Department of Psychology, University of Salzburg, Hellbrunnerstrasse 34, 5020, Salzburg, Austria. Fax: $+43 /(0) 662 /$ 80445126.

E-mail: martina.goslar@sbg.ac.at

\begin{abstract}
Background and aims: Internet addiction, sex addiction and compulsive buying are common behavioral problems, which share similarities with gambling disorder and substance use disorders. However, little is known about the efficacy of their treatments. The objective of this meta-analysis was to examine the efficacy of the treatments of such problem behaviors, and to draw parallels to gambling disorder and substance use disorders in terms of treatment response. Methods: Literature search yielded 91 studies totaling 3,531 participants to provide a comprehensive evaluation of the short-term and long-term efficacy of psychological, pharmacological and combined treatments for internet addiction, sex addiction, and compulsive buying. Results: Psychological, pharmacological, and combined treatments were associated with robust prepost improvements in the global severity of internet addiction (Hedges's g: 1.51, 1.13, and 2.51, respectively) and sex addiction (Hedges's g: 1.09, 1.21, and 1.91, respectively). For compulsive buying, psychological and pharmacological treatments were also associated with a large-sized pre-post reduction in global severity (Hedges's g: 1.00 and 1.52, respectively). The controlled pre-post and within-group pre-follow-up effect sizes were in the similar range, with few exceptions. Moderator analyses suggest that psychological interventions are effective for reducing compulsive behaviors, especially when delivered face-to-face and conducted over extended periods of time. Combinations of cognitive-behavioral approaches with medications showed an advantage over monotherapies. Discussion and Conclusions: The results suggest that treatments for common behavioral addictions are effective in the short term, similar to those implemented for gambling disorder and substance use disorders, but more rigorous clinical trials are needed.
\end{abstract}

\section{KEYWORDS}

Internet addiction, sex addiction, compulsive buying, treatment, meta-analysis

Recent research has identified similarities between substance use disorders (SUDs) and behavioral addictions (BAs; e.g., Grant, Potenza, Weinstein, \& Gorelick, 2010). Accordingly, non-substance related behavioral addictions had been defined based on the criteria for substance use specified in the Diagnostic and Statistical Manual of Mental Disorders (DSM IV; American Psychiatric Association, 1994) including preoccupation with the specific behavior, lack of control over the behavior, tolerance, withdrawal, and continued behavior despite negative consequences (e.g., Grant et al., 2010). Currently, only gambling disorder (GD), which was subsumed under "Impulse-Control Disorders Not Elsewhere Classified" in the DSM IV (American Psychiatric Association, 1994), is categorized under the new section "Substancerelated and Addictive Disorders" of the DSM-5 (American Psychiatric Association, 2013). This reorganization has stimulated much debate as to whether further behaviors with diminished impulse control should be considered as possible candidates for BAs (e.g., Grant et al., 2010; Mueller et al., 2019). 
Apart from GD, internet gaming disorder (IGD) is the only condition that is placed in the DSM-5 under Section III with the recommendation for further research (American Psychiatric Association, 2013). Supported by experts from various clinical and public health domains (e.g., Rumpf et al., 2018; Saunders et al., 2017), gaming disorder is also considered in the draft of the ICD-11 (World Health Organization, 2018). It is important to note that IGD should be distinguished from the global designation internet addiction (IA), as both represent different constructs (e.g., Griffiths \& Pontes, 2014; Kiraly et al., 2014). However, since many publications refer to global IA, this term has also been adopted in this paper. Moreover, a distinction should be made between "gaming" and "gambling": Whereas "gaming is principally defined by its interactivity, predominantly skill-based play, and contextual indicators of progression and success, ... gambling is defined by betting and wagering mechanics, predominantly chance-determined outcomes, and monetisation features that involve risk and payout to the player." (King, Gainsbury, Delfabbro, Hing, \& Abarbanel, 2015, p. 216).

Although the inclusion of IGD in the diagnostic manuals is discussed controversially in the scientific literature (King et al., 2019; Petry, Rehbein, Ko, \& O’Brien, 2015; Rumpf et al., 2018; Saunders et al., 2017), much research has already been done on IA and IGD, particularly on neurobiological measures suggesting parallels to SUDs (for reviews see Fauth-Buhler \& Mann, 2017; Kuss, Pontes, \& Griffiths, 2018). Aside from similarities between SUDs and BAs in terms of phenomenological and clinical characteristics, comorbidity and family history, particularly findings from neuroscience research appear essential for identifying indicators of addictive behaviors (e.g., Grant et al., 2010; Potenza, Sofuoglu, Carroll, \& Rounsaville, 2011).

In line with this consideration, some progress in the examination of neurobiological commonalities with SUDs has been achieved more recently within the domains of sex addiction (SA) and compulsive buying (CB) by analyzing phenomena traditionally investigated in SUDs such as conditioning processes (e.g., Hoffmann, Goodrich, Wilson, \& Janssen, 2014; Snagowski, Laier, Duka, \& Brand, 2016), cue reactivity, attentional bias and related neural network activation (e.g., Brand, Snagowski, Laier, \& Maderwald, 2016; Gola et al., 2017; Jiang, Zhao, \& Li, 2017; Laier, Pawlikowski, \& Brand, 2014; Laier, Schulte, \& Brand, 2013; Lawrence, Ciorciari, \& Kyrios, 2014; Mechelmans et al., 2014; Pekal, Laier, Snagowski, Stark, \& Brand, 2018; Schmidt et al., 2017; Seok \& Sohn, 2015; Starcke, Schlereth, Domass, Schöler, \& Brand, 2012; Trotzke, Starcke, Pedersen, \& Brand, 2014; Trotzke, Starcke, Pedersen, Müller, \& Brand, 2015; Voon et al., 2014), or executive functioning (Derbyshire, Chamberlain, Odlaug, Schreiber, \& Grant, 2014; Messina, Fuentes, Tavares, Abdo, \& Scanavino, 2017; Raab, Elger, Neuner, \& Weber, 2011; Trotzke et al., 2015). These studies demonstrated that among those conditions that have not yet been officially recognized in the DSM-5 as BAs, the currently available evidence regarding neurobiological indicators for parallels between substance-related and non substance-related behaviors mainly comes from the areas of IA, SA and CB, which are the focus of the present paper. Since these problems are of clinical relevance, and often associated with harmful consequences for affected individuals (e.g., Pontes, Kuss, \& Griffiths, 2015), effective treatment options need to be investigated (e.g., Grant et al., 2010). To date, published meta-analyses have primarily been conducted with respect to IA proving the efficacy of various treatment approaches (Chun, Shim, \& Kim, 2017; Liu, Liao, \& Smith, 2012; Winkler, Doersing, Rief, Shen, \& Glombiewski, 2013). Two of the meta-analyses examined psychological, pharmacological and combinations of both interventions, but the evidence was limited to treatment outcome studies in China (Liu et al., 2012), and Korea (Chun et al., 2017). The most comprehensive meta-analytic review supported the evidence for the efficacy of psychotherapy and medical treatments to reduce symptoms of IA including trials from Asian and western countries (Winkler et al., 2013). Combined interventions, however, were not considered. Moreover, the meta-analysis of Winkler et al. (2013) did not include more recent research.

Favorable results for psychological and pharmacological interventions in reducing global severity of $\mathrm{CB}$ were also found in another recent meta-analysis (Hague, Hall, \& Kellett, 2016). However, the impact of study quality and other moderators on treatment outcomes was not examined. Consequently, a comprehensive investigation of treatment options for IA and $\mathrm{CB}$ is still pending. Although SA is considered in the ICD-11 with the term "compulsive sexual behaviour disorder" (World Health Organization, 2018), and "self-reported feelings of addiction to pornography are not uncommon" (Grubbs, Kraus, \& Perry, 2019, p. 93), treatments for SA have not yet been examined by metaanalytic methods. Additionally, no comparisons have yet been made between IA, or IGD-a candidate for the section "Substance-related and Addictive Disorders" of the DSMand other possibly addictive behaviors, such as SA and CB, based on treatment response, which is considered an important indicator for parallels between SUDs and BAs (e.g., Grant et al., 2010).

The primary objective of the present meta-analysis, therefore, was to investigate the efficacy of psychological, pharmacological and combined psychological and pharmacological interventions for IA, SA, and CB for reducing (a) the global severity and (b) the frequency of compulsive behaviors after treatment cessation (short-term effects) and at the last reported follow-up period (long-term effects). Based on findings in recent reviews (Hague et al., 2016; Winkler et al., 2013), we expected psychological and pharmacological treatments to be equally effective across the three addiction categories. We further expected that treatment outcomes are similar to those reported for substance use and gambling (Grant et al., 2010; Potenza et al., 2011). In addition, our goal was to identify potential moderators of the effect sizes within each addiction category. The meta-analysis was conducted according to the recommendations of the PRISMA Statement (Moher, Liberati, Tetzlaff, \& Altman, 2009). 


\section{METHODS}

\section{Eligibility criteria}

Studies were considered for inclusion if they (1) employed any kind of psychological, pharmacological, or combined intervention (e.g., psychological and pharmacological interventions applied at the same time); (2) used within-group, randomized, or quasi-randomized controlled study designs including wait-list controls, participants not receiving treatment, alternative active treatments, or a placebo intervention; (3) treated participants with the diagnosis of IA, SA, or CB; (4) measured at least one of the outcome variables (i.e., global severity or frequency); and (5) reported sufficient statistical data for effect size calculations. Studies were excluded if (1) the study was a single case study; (2) the study sample overlapped completely with the sample of another study included in the meta-analysis; (3) the treatment was not described, or (4) no abstract or full text of the study was available. With respect to SA, we only included studies investigating excessive sexual behaviors following the definition proposed by Kafka (2010), and excluded studies focusing treatments of paraphilias that differ from SA in terms of "socially anomalous or 'deviant' forms of sexual preference” (Kafka, 2010, p. 392).

\section{Information sources and literature search}

We conducted a multilevel literature search using the databases PsycInfo, Medline, PubMed, Psyndex, and ISI Web of Knowledge. The search covered all relevant publications from the first available year until June 30, 2019 using the following disorder-related search terms: Internet addiction, online addict*, internet gaming disorder, online game addict*, video game addict*, videogame addict*, computer game addict*, smartphone addict*, mobile phone addict*, social media addict*, facebook addict*, problem* cellular phone; sex* addict*, sex* compuls*, sex* impuls*, hypersex*, nonparaphilic sex*, paraphilia-related disorder*; compulsive shopping, impulsive buy*, oniomania, shopaholic*, overshopping combined with the interventionrelated key words treatment, intervention, therapy, psychotherapy. The same search terms were used to browse ProQuest Digital Dissertations for unpublished, gray literature. Subsequently, we conducted a thorough examination of the reference lists of review articles, meta-analyses, and original studies retrieved from the databases. Additionally, authors of relevant articles were contacted to ask for missing data and/ or unpublished papers suitable for inclusion in the metaanalysis. Chinese publications were translated by two native speakers with an academic background.

\section{Outcome measures}

Following the most commonly reported outcome measures in the original studies, we specified two outcome variables to determine the reduction of pathological symptoms: (1) the global severity, quantified by the use of relevant assessment tools, and (2) the frequency (e.g., number of hours spent online, pornography viewing, or the number of buying episodes in the last week or month), quantified by diary cards or self-reports.

\section{Study selection}

Study selection was performed by two independent reviewers (the first and the second authors, MG and ML), and supervised by the last author of this paper (AL). Disagreements between the authors were resolved through discussion.

\section{Data collection process and data extraction}

We generated a structured data extraction form that we refined and modified after pilot testing a sample of 10 studies. To calculate pre-post and pre-follow-up withingroup effect sizes, numerical data were extracted for each treatment condition and outcome separately. If different psychological or pharmacological treatments were examined within one study, data for each condition were recorded separately and included in the within-group effect sizes for statistical analyses. To calculate pre-post controlled effect sizes, data from wait-list, no treatment, and placebo control groups were included. Additionally, we extracted numerical and categorical data from each study in order to perform moderator analyses. Data extraction was performed by the first author (MG), and validated by the second author (ML). The ratings of the two independent coders focused on the types of treatments, the measurement of the outcome variables, and the reliability and validity of the disorder-specific diagnoses. In the studies, however, the same tools have been applied both for the assessment of disorder-specific diagnoses and the measurement of the outcome variable "global severity" during treatment. Because the rating of the reliability and validity of the tools used for the measurement of the outcome variables was also part of the rating of the risk of bias in individual studies (see below), the interrater reliability quantified by the kappa statistic was performed only for the types of treatments.

\section{Risk of bias in individual studies}

We assessed the internal validity of each study using the Quality Assessment Tool for Quantitative Studies, developed by the Effective Public Health Practice Project (EPHPP) (Thomas, Ciliska, Dobbins, \& Micucci, 2004). This tool has demonstrated content and construct validity (Thomas et al., 2004) and is recommended for systematic reviews and metaanalyses (Deeks et al., 2003). Each study was rated in a standardized manner on six domains: selection bias, study design, identification and control of confounders, blinding, reliability and validity of data collection tools, and reporting and percentage of withdrawals and dropouts. Each domain was evaluated as strong, moderate, or weak. The global rating was calculated after evaluation of the six domains. The first two authors (MG and ML) independently assessed each study and determined the global score of each trial. Interrater reliability was quantified using the kappa statistic. 
Disagreements between the authors were resolved through discussion until consensus was reached.

\section{Effect size calculation and quantitative data synthesis}

Statistical analysis was performed using the software program Comprehensive Meta-Analysis (CMA) version 2.2.064 (Borenstein, Hedges, Higgins, \& Rothstein, 2005). Within each addiction category, we calculated effect sizes for the outcome variables reported in psychological, pharmacological, and combined studies separately for within-group and controlled study designs (see Appendix for formulas). Due to small sample sizes, the effect sizes were corrected for bias using Hedges's $g$ with the corresponding $95 \%$ confidence interval (CI; Hedges \& Olkin, 1984). If means and standard deviations were not available, effect sizes were calculated based on equivalent estimation procedures (e.g., $t$ values, or exact probability levels). If an outcome variable was measured by more than one instrument, data from these instruments were entered separately and pooled together for the particular outcome variable (Lipsey \& Wilson, 2000). For studies reporting data based on both completers and intention-to-treat (ITT) analyses, the ITT data was taken into account. The direction of the effect was adjusted according to the "success": the effect size was positive if the treated group performed superior to the control group. According to Cohen's recommendations (1977), effect sizes of 0.20 to 0.30 can be classified as small, those near 0.50 as medium, and those above 0.80 as large.

Assuming heterogeneity among the studies, we decided to use the random effects model for the integration of effect sizes. Heterogeneity of the effect sizes was investigated using the Q statistic with the corresponding $p$ value, and the $I^{2}$ statistic, indicating to what extent real differences in effect sizes was reflected by the proportion of the variance (Borenstein, Hedges, Higgins, \& Rothstein, 2009; Higgins, Thompson, Deeks, \& Altman, 2003); $I^{2}$ values of $25 \%, 50 \%$, and $75 \%$ were classified as low, moderate, and high, respectively (Higgins et al., 2003).

\section{Risk of bias across studies}

To control for publication bias, we conducted a thorough literature search and computed Rosenthal's fail-safe $N$ (Rosenthal, 1979) and also examined funnel plots (Duval \& Tweedie, 2000). According to Rosenthal (1991), effect sizes are considered robust if the number of studies needed to obtain a nonsignificant overall effect is greater than $5 k+10$, where $k$ represents the number of studies. Additionally, we used the trim-and-fill method (Duval \& Tweedie, 2000) to estimate missing studies and their impact on the ascertained effect sizes. This method is based on the logic of the funnel plot and assumes a symmetrical distribution of the effect sizes for outcome variables in the absence of publication bias. In the case of asymmetrical distribution, the trim-andfill method adjusts and corrects the effect sizes (Borenstein et al., 2009); we only applied this method if 10 studies were available for the analysis (Sterne, Egger, \& Moher, 2011). Funnel plot asymmetry was assessed by using Egger's test
(Egger, Smith, Schneider, \& Minder, 1997). As singular extreme effect size values produce misleading interpretations of treatment effects (Lipsey \& Wilson, 2000), we used the "one-study-removed" method offered by CMA to examine the impact of each study's effect size on the overall effect (Borenstein et al., 2005). If the recalculated results did not substantially impact the effect size and remained within the 95\% CI, studies were retained in the analyses.

\section{Moderator analysis}

To explain heterogeneity among effect sizes, we examined the type of data analysis (ITT vs. completer analysis), and the quality of studies (EPHPP global scores) as possible moderators. Because depression and anxiety were found to be associated with BAs (e.g., González-Bueso et al., 2018; Starcevic \& Khazaal, 2017), we examined whether the effect sizes varied as a function of these co-occurring disorders (inclusion vs. exclusion of depression and/or anxiety). Since co-occurring disorders, particularly depression and anxiety, are most common among individuals affected by BAs (Starcevic \& Khazaal, 2017), studies that failed to report data on comorbid conditions were assumed to include participants with cooccurring depression and anxiety as well. For psychological studies, we further investigated the mode of treatment (group setting vs. individual counseling vs. other types of settings [e.g., individual and group setting, family setting]), the mode of delivery (face-to-face [FTFTs] vs. self-guided treatments [SGTs]), and the type of psychological intervention. The type of psychological intervention was analyzed by dividing psychological strategies into the following subcategories: (1) CBT, covering cognitive and/or behavioral treatments; (2) integrative treatment involving a variety of different treatment approaches, and (3) psychological therapies that pertain to other categories, such as family therapy, reality therapy, acceptance and commitment therapy, or art therapy. Assuming that a number of studies were conducted in non-western countries, particularly for IA, we followed a previous meta-analysis (Winkler et al., 2013) and explored whether the cultural background (Asian vs. other countries) proved to be a moderator. Since global IA and IGD represent different constructs (e.g., Griffiths \& Pontes, 2014), we also examined the differences between studies based on global IA and those, that investigated IGD and other internet-enabled activities (e.g., smartphone addiction, videogame addiction).

For pharmacological treatments, we examined whether antidepressants performed superior to other types of medications or to mixed medications (e.g., antidepressants combined with methylphenidate). For combined studies, we examined both the impact of the types of psychological and pharmacological interventions. Additionally, we investigated whether one of the types of treatment (psychological vs. pharmacological vs. combined interventions) within each addiction category showed an advantage over the others. Finally, we compared the effect sizes of psychological and pharmacological interventions of the different addiction categories. Accounting for the fact that "the internet is just a channel through which individuals may access whatever 
content they want (e.g., gambling, shopping, chatting, sex)" (Griffiths \& Pontes, 2014, p. 2), we subsumed studies that included individuals with excessive sexual or buying behaviors under the categories "sex addiction" and "compulsive buying", regardless of whether the internet was used or not.

Moderator analyses for categorical variables were conducted using the mixed effects model with pooled estimates of $T^{2}$ and the Q-test based on analysis of variance with the corresponding $p$ value for the interpretation of the differences between subgroups (Borenstein et al., 2009). In the case of at least 10 available studies (Deeks, Higgins, \& Altman, 2011), we further conducted meta-regression analyses using the year of publication and the duration of treatment (assessed with the total number of hours spent in treatment in psychological trials, or with the number of weeks in pharmacological trials). If an insufficient number of psychological studies indicated the number of hours spent in treatment, the number of weeks was used to measure treatment duration. Meta-regression analyses on the mean age and the percentage of male/female participants were not performed because age and sex across studies differ from that within studies hampering reliable interpretation (Thompson \& Higgins, 2002).

\section{RESULTS}

\section{Study selection}

The flow diagram of the study selection process is illustrated in Fig. 1. There were no interrater disagreements regarding the types of treatments.

\section{Characteristics of studies, treatments, and participants}

Across all addiction categories, the present sample of studies varied in type of control condition: Half of them implemented no control group (50\%) and several studies used waitlist, no treatment, healthy controls, or placebo control groups $(30 \%)$, or other active treatment comparisons $(20 \%)$. Results were mainly based on completers (80\%). Follow-up data were provided by 32 psychological studies (IA: $k=16$ studies with periods ranging from 1 to 6 months; $M=3.53$, $S D=2.13$; SA: $k=11$ studies with periods ranging from 1.5 to 6 months; $M=4.27, S D=1.88$; CB: $k=5$ studies with periods ranging from 3 to 6 months; $M=5.4, S D=1.34$ ), by one pharmacological study in the CB category with 12 months follow-up, and by two studies in the IA category which used combined interventions, each collecting data at one month follow-up.

The majority of psychological studies examined CBT (58\%), delivered treatment through group settings (71\%), and in face-to-face format (92\%). The total number of hours spent in psychological interventions ranged from 15 min to $54 \mathrm{~h}(M=12.55 \mathrm{~h}, S D=10.49)$, from one week to 26 weeks $(M=10.44, S D=6.12)$, and from 8 weeks to 20 weeks $(M=11.71, S D=3.90)$ for the treatment of IA, SA, and $\mathrm{CB}$, respectively. Most pharmacological studies examined antidepressants (85\%); the majority of combined trials used CBT in combination with antidepressants (71\%). The duration of pharmacological treatments ranged from 6 to 52 weeks $(M=15.67, S D=17.95)$, from 12 to 72 weeks $(M=24.83, S D=23.58)$, and from 7 to 12 weeks $(M=9.50, S D=2.20)$ for the treatment of IA, SA, and CB, respectively.

\begin{tabular}{|c|c|c|c|c|c|c|c|}
\hline \multicolumn{3}{|c|}{ Internet Addiction } & \multicolumn{3}{|c|}{ Sex Addiction } & \multicolumn{2}{|c|}{ Compulsive Buying } \\
\hline \multicolumn{3}{|c|}{$\begin{array}{l}\text { Articles identified by literature search }(k=2,113) \text { : } \\
\text { PsycInfo }(k=541) ; \text { Medline }(k=292) ; \text { PubMed }(k= \\
\text { 439); Pyndex }(k=4308) ; \text { ISI }(k=247) ; \text { ProQuest }(k= \\
\text { 257); reviews and primary studies }(k=194)\end{array}$} & \multicolumn{3}{|c|}{$\begin{array}{l}\text { Articles identified by literature search }(k=4,163): \\
\text { PsycInfo }(k=1,094) ; \text { Medline }(k=426) ; \text { PubMed }(k= \\
1,052) ; \text { Pyndex }(k=48) ; \text { ISI }(k=590) ; \text { ProQuest }(k= \\
869) ; \text { reviews and primary studies }(k=84)\end{array}$} & \multicolumn{2}{|c|}{$\begin{array}{l}\text { Articles identified by literature search }(k=1,107): \\
\text { PsycInfo }(k=256) ; \text { Medline }(k=183) ; \text { PubMed }(k=236) ; \\
\text { Pyndex }(k=41) ; \text { ISI }(k=75) ; \text { ProQuest }(k=202) ; \\
\text { reviews and primary studies }(k=114)\end{array}$} \\
\hline & & \\
\hline \multicolumn{3}{|c|}{ Articles after duplicates removed $(k=1,464)$} & \multicolumn{3}{|c|}{ Articles after duplicates removed $(k=3,269)$} & \multicolumn{2}{|c|}{ Articles after duplicates removed $(k=715)$} \\
\hline & & \\
\hline \multicolumn{3}{|c|}{$\begin{array}{l}\text { Articles excluded }(k=1,383) \text { : } \\
\text { No internet addiction }(k=433) \\
\text { Reviews/qualitative }(k=364) \\
\text { Treatment not targeted }(k=524) \\
\text { No psychological/pharmacological intervention }(k=3) \\
\text { Case studies }(k=42) \\
\text { No full text available }(k=17)\end{array}$} & \multicolumn{3}{|c|}{$\begin{array}{l}\text { Articles excluded }(k=3,237) \\
\text { No sex addiction }(k=2,419) \\
\text { Reviews/qualitative }(k=529) \\
\text { Treatment not targeted }(k=234) \\
\text { No psychological/pharmacological intervention }(k=0) \\
\text { Case studies }(k=55) \\
\text { No full text available }(k=0)\end{array}$} & \multicolumn{2}{|c|}{$\begin{array}{l}\text { Articles excluded }(k=699) \text { : } \\
\text { No compulsive buying }(k=421) \\
\text { Reviews/qualitative }(k=125) \\
\text { Treatment not targeted }(k=134) \\
\text { No psychological/pharmacological intervention }(k=1) \\
\text { Case studies }(k=18) \\
\text { No full text available }(k=0)\end{array}$} \\
\hline & \multirow{2}{*}{\multicolumn{3}{|c|}{ Articles selected for further screening $(k=32)$}} & \\
\hline \multicolumn{3}{|c|}{ Articles selected for further screening $(k=81)$} & & & & \multicolumn{2}{|c|}{ Articles selected for further screening $(k=16)$} \\
\hline \multicolumn{3}{|c|}{ Articles selected for further screening $(k=81)$} & \multicolumn{3}{|c|}{$\downarrow$} & \multicolumn{2}{|c|}{ 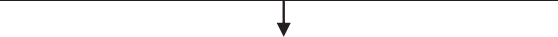 } \\
\hline \multicolumn{3}{|c|}{$\begin{array}{l}\text { Articles excluded }(k=26): \\
\text { No primary outcome variable }(k=10) \\
\text { Treatment not described }(k=3) \\
\text { Sample overlap }(k=4) \\
\text { Insufficient statistical data }(k=9)\end{array}$} & \multicolumn{3}{|c|}{$\begin{array}{l}\text { Articles excluded }(k=9) \\
\text { No primary outcome variable }(k=1) \\
\text { Treatment not described }(k=0) \\
\text { Sample overlap }(k=3) \\
\text { Insufficient statistical data }(k=5)\end{array}$} & \multicolumn{2}{|c|}{$\begin{array}{l}\text { Articles excluded }(k=3) \\
\text { No primary outcome variable }(k=0) \\
\text { Treatment not described }(k=0) \\
\text { Sample overlap }(k=2) \\
\text { Insufficient statistical data }(k=0) \\
\text { Data from post to follow-up only reported }(k=1)\end{array}$} \\
\hline & & \multirow{2}{*}{\multicolumn{2}{|c|}{ Studies included in the meta-analysis $(k=13)$}} \\
\hline \multicolumn{3}{|c|}{ Studies included in the meta-analysis $(k=55)$} & \multicolumn{3}{|c|}{ Studies included in the meta-analysis $(k=23)$} & & \\
\hline & & & $\downarrow$ & & + & & \\
\hline $\begin{array}{l}\text { Psychological } \\
\text { studies }(k=43) \\
\text { including } 54 \\
\text { treatment } \\
\text { conditions }\end{array}$ & $\begin{array}{l}\text { Pharmacological } \\
\text { studies }(k=6) \\
\text { including } 8 \\
\text { treatment } \\
\text { conditions }\end{array}$ & $\begin{array}{l}\text { Combined } \\
\text { studies }(k=6) \\
\text { Including } 7 \\
\text { treatment } \\
\text { conditions }\end{array}$ & $\begin{array}{l}\text { Psychological } \\
\text { studies }(k=16) \\
\text { including } 18 \\
\text { treatment } \\
\text { conditions }\end{array}$ & $\begin{array}{l}\text { Pharmacological } \\
\text { studies }(k=5)\end{array}$ & $\begin{array}{l}\text { Combined } \\
\text { studies }(k=2)\end{array}$ & $\begin{array}{l}\text { Psychological studies }(k=6) \\
\text { including } 7 \text { treatment } \\
\text { conditions }\end{array}$ & $\begin{array}{l}\text { Pharmacological studies } \\
(\mathrm{k}=7)\end{array}$ \\
\hline
\end{tabular}

Figure 1. Flow diagram of the study selection process 
Across all addiction categories, a total of 3,531 participants were analyzed (IA: $n=2,427$; SA: $n=771$; CB: $n=333)$. The majority of the studies included participants with co-occurring depression and anxiety (77\%). Trials focusing on IA were mainly conducted in Asian countries (75\%). The total sample was predominantly male in studies examining IA (76\%) with an average age of 21 , and SA (98\%) with an average age of 37 , but female in studies examining CB (92.45\%) with an average age of 42 . Detailed information regarding the characteristics of studies is presented in Tables $1-3$.

\section{Risk of bias within studies}

The global EPHPP scores for the studies included in the different addiction categories are outlined in the Tables 1-3. Validity assessment was conducted by two independent raters yielding an interrater reliability of $\kappa=0.73$ for studies in the IA and SA categories, and $\kappa=0.75$ for studies in the $\mathrm{CB}$ category.

\section{Synthesis of results and risk of bias across studies}

The pooled effect sizes for all types of addictions and treatments separately for within-group and controlled study designs on all outcomes at posttreatment and follow-up, the 95\% CI, and the significance tests are outlined in Table 4. The forest plots on the within-group effect sizes for each condition, treatment, and outcome at posttreatment are presented in Fig. 2.

\section{Effect sizes for psychological treatments at posttreatment and follow-up}

Psychological treatments across addiction categories yielded short-term effect sizes ranging from medium to large in both study designs. Long-term effect sizes in all addiction categories indicated that treatment effects were maintained. As depicted in Table 4, predominantly high heterogeneity across studies was observed for the outcome variables within the IA and SA categories, and moderate heterogeneity or homogeneity was observed in the $\mathrm{CB}$ category.

Within the IA category, the trim-and-fill method identified 17 studies causing funnel plot asymmetry for the reduction of global severity and one study for the reduction of frequency in within-group study designs. The analyses with these filled studies suggested slightly reduced effect sizes (global severity: $g=0.87$; 95\% CI [0.82, 0.92]; Egger's test $p<0.001$; frequency: $g=0.93$; 95\% CI [0.84, 1.03]; Egger's test $p=0.282$ ) suggesting a nonsignificant impact of publication bias. No indication for publication bias was found for the reduction of global severity based on controlled study designs (Egger's test $p=0.067$ ). Within the SA category, the trim-and-fill method identified one study causing funnel plot asymmetry for the reduction of global severity leading to a slightly reduced effect size for this outcome variable ( $g=0.88 ; 95 \%$ CI $[0.79 ; 0.97]$, Egger's test $p=0.318)$. Whenever fail-safe $N$ analyses were conducted, the effect sizes across all addiction categories were considered robust for the outcome variables, except for the controlled effect size regarding the reduction of global severity in the SA and CB categories, which were not robust.

\section{Effect sizes of pharmacological treatments at posttreatment and follow-up}

At posttreatment, the within-group effect sizes across all addiction categories were medium and large. Controlled effect sizes were mainly based on single trials ranging from large in the IA category to small and negative in the SA and $\mathrm{CB}$ categories. The lack of follow-up data precluded the interpretation of long-term effect sizes. High and moderate heterogeneity across studies was observed for the outcome variables within the addiction categories. The fail-safe $N$ analyses performed for the available data suggested the robustness of the effect sizes.

\section{Effect sizes of combined treatments at posttreatment and follow-up}

Combined interventions were implemented only for the treatment of IA and SA based on within-group study designs yielding large short-term effect sizes. Follow-up data were available only in the IA category producing equally large effect sizes. High heterogeneity across the studies was observed for the reduction of global severity in the IA category; however the fail-safe $N$ indicated the robustness of the effect size.

Outlier identification through the one-study-removed procedure showed no impact of any single study on the overall effects for psychological, pharmacological, and combined treatments.

\section{Moderator analyses}

Moderator analyses were conducted for within-group effect sizes. The results for categorical variables at posttreatment are presented in Table 5.

Effect sizes across all types of addictions and interventions remained unaffected by the quality of studies, co-occurring depression and anxiety, and the year of publication (IA: global severity: $\beta=-0.02 ; \mathrm{SE}=0.03 ; p=0.417$; frequency: $\beta=-0.09 ; \mathrm{SE}=0.05 ; p=0.075$; SA: global severity: $\beta=-0.03$; $\mathrm{SE}=0.04 ; p=0.519$ ).

In regards to IA, significantly larger effect sizes were found for FTFTs compared to SGTs, and for interventions including a higher number of hours in treatment for the reduction of global severity $(\beta=0.04$; $\mathrm{SE}=0.01 ; p<0.01)$ and frequency $(\beta=0.03$; SE $=0.009 ; p<0.01)$. For the reduction of global severity in pharmacological studies, larger effect sizes emerged for antidepressants compared to other chemical agents (i.e., methylphenidate, atomoxetine), for those implemented in other countries compared to Asian countries, and examining global IA compared to IGD and smart phone addiction.

With respect to SA, CBT and other psychological treatments (i.e., acceptance and commitment therapy) showed an advantage over integrative interventions and of individual counseling 
Table 1. Characteristics of studies for internet addiction

\begin{tabular}{|c|c|c|c|c|c|c|c|c|}
\hline Study/Year & $\begin{array}{cc}\text { Treatment group }(N) / \text { Mode of } \\
N^{\mathrm{a}} \quad \text { therapy/Mode of delivery }^{\mathrm{b}}\end{array}$ & $\begin{array}{l}\text { Control group }(N) / \text { Mode of } \\
\text { therapy/Mode of delivery }\end{array}$ & $\begin{array}{l}\text { Culture/D/A } \\
(+/-) / \text { IA type }\end{array}$ & $\underset{c^{c}}{\text { Duration } t /}$ & $\begin{array}{c}\mathrm{FU} \\
\text { (months) }\end{array}$ & $\begin{array}{l}\text { Outcomes } \\
\text { (assessment) }\end{array}$ & $\begin{array}{l}\text { Data } \\
\text { analysis }\end{array}$ & ЕРНРP \\
\hline \multicolumn{9}{|c|}{ Psychological treatments } \\
\hline $\begin{array}{l}\text { Anuradha and } \\
\text { Singh }(2018)\end{array}$ & 28 CBT (28)/I/FTFT & None & Asia/-/IA & NA & None & GS (IADQ) & $\mathrm{CO}$ & 3 \\
\hline Bai and Fan (2007) & $\begin{array}{l}48 \text { IT (CBT; self-control; } \\
\text { social competence) } \\
(24) / \mathrm{G} / \mathrm{FTFT}\end{array}$ & NT (24) & Asia/+/IA & 16 & 1.5 & GS (CIAS-R) & $\mathrm{CO}$ & 3 \\
\hline Cao et al. (2007) & 57 CBT (26)/G/FTFT & NT (31) & Asia/+/IA & 10 & None & GS (YDQ, CIAS) & $\mathrm{CO}$ & 2 \\
\hline Celik (2016) & $30 \mathrm{EDU}(15) / \mathrm{G} / \mathrm{FTFT}$ & NT (15) & Turkey/+/IA & 10 & 6 & $\begin{array}{l}\text { GS (PIUS)FR (\% of } \\
\text { Internet game } \\
\text { playing among } \\
\text { Internet use } / \mathrm{w})^{\mathrm{d}}\end{array}$ & NA & 3 \\
\hline Deng et al. (2017) & 63 CBI (44)/G/FTFT & WL (19) & Asia/+/IGD & 18 & 6 & GS (CIAS) & $\mathrm{CO}$ & 2 \\
\hline Du et al. (2010) & $\begin{array}{l}56 \text { IT (CBT; parent training; } \\
\text { EDU for teachers) } \\
\text { (32)/G/FTFT }\end{array}$ & NT (24) & Asia/+/IA & 14 & 6 & GS (IOSRS) & $\mathrm{CO}$ & 2 \\
\hline $\begin{array}{l}\text { González-Bueso } \\
\text { et al. (2018) }\end{array}$ & $\begin{aligned} 30 \text { 1) } & \text { CBT (15)/I/FTFT2) IT } \\
& (\mathrm{CBT}+\mathrm{EDU} \text { for parents) } \\
& (15) / \mathrm{I} / \mathrm{FTFT}\end{aligned}$ & $\mathrm{HC}(30)^{\mathrm{e}}$ & Spain/-/IGD & 1) 9 & None & GS (DQVMIA) & $\mathrm{CO}$ & 3 \\
\hline Guo et al. (2008) & 28 1) CBT (14)/G/FTFT & $\begin{array}{l}\text { 2) SUPP (e.g., sharing } \\
\text { information on IA; } \\
\text { promotion of self-esteem } \\
\text { and resources) } \\
(14) / G / \text { FTFT }^{f}\end{array}$ & Asia/+/IA & $\begin{array}{l}\text { 1) } 8 \\
\text { 2) } \mathrm{NA}\end{array}$ & None & GS (CIAS) & $\mathrm{CO}$ & 2 \\
\hline Han et al. (2012) & $14 \mathrm{FT} \mathrm{(14)/F/FTFT}$ & None & Asia/-/IGD & NA & None & GS (YIAS)FR (h/w) & $\mathrm{CO}$ & 3 \\
\hline Han et al. (2018) & 26 CBT (26)/G/FTFT & None & Asia/-/IGD & 24 & None & GS (CIAS)FR (h/w) & $\mathrm{CO}$ & 3 \\
\hline Hui et al. (2017) & 73 1) CBT (37)/G/FTFT & $\begin{array}{l}\text { 2) } \mathrm{IT}(\mathrm{CBT}+\mathrm{EA}) \\
(36) / \mathrm{I}+\mathrm{G} / \mathrm{FTFT}^{\mathrm{f}}\end{array}$ & Asia/-/IGD & $\begin{array}{l}\text { 1) } 5 \\
\text { 2) } 10\end{array}$ & None & GS (IAD) & $\mathrm{CO}$ & 2 \\
\hline Ke and Wong (2018) & 157 CBT (157)G/FTFT & None & Asia/+/IA & 12 & 1 & GS (PIUQ) & $\mathrm{CO}$ & 3 \\
\hline Khazaei et al. (2017) & 48 PI (24)/G/FTFT & WL (24) & Iran/+/IA & NA & None & GS (IAT)FR (h/w) & NA & 3 \\
\hline Kim (2008) & 25 RT (13)/G/FTFT & NT (12) & Asia/+/IA & 12.5 & None & GS (K-IAS) & NA & 3 \\
\hline King et al. (2017) ${ }^{\mathrm{g}}$ & $\begin{array}{l}\text { CBT ( } 84 \mathrm{~h} \text { abstinence) } \\
(9) / \mathrm{I} / \mathrm{NA}\end{array}$ & None & $\begin{array}{l}\text { Australia/ } \\
\text { +/IGD }\end{array}$ & NA & 1 & $\begin{array}{l}\text { GS (IGD checklist) } \\
\text { FR }(\mathrm{h} / \mathrm{w})\end{array}$ & $\mathrm{CO}$ & 3 \\
\hline Lan et al. (2018) & 54 1) $\mathrm{CBT}(27) / \mathrm{G} / \mathrm{FTFT}$ & 2) $\mathrm{EDU}(27) / \mathrm{G} / \mathrm{FTFT}^{\mathrm{f}}$ & Asia/+/SMA & 1) 8 & 3 & GS (MPIAS)FR (h/w) & $\mathrm{CO}$ & 2 \\
\hline Lee et al. (2016) & $\begin{array}{l}46 \text { CBT (home-based daily } \\
\text { writing) (46)/FTFT/I }\end{array}$ & None & Asia/+/SMA & NA & None & GS (KSAPS) & $\mathrm{CO}$ & continued) \\
\hline
\end{tabular}


Table 1. Continued

\begin{tabular}{|c|c|c|c|c|c|c|c|c|}
\hline Study/Year & $\begin{array}{l}\text { Treatment group }(N) / \text { Mode of } \\
\text { therapy/Mode of delivery }{ }^{\mathrm{b}}\end{array}$ & $\begin{array}{l}\text { Control group }(N) \text { /Mode of } \\
\text { therapy/Mode of delivery }\end{array}$ & $\begin{array}{l}\text { Culture/D/A } \\
(+/-) / \text { IA type }\end{array}$ & $\begin{array}{c}\text { Duration } t / \\
c^{c}\end{array}$ & $\begin{array}{c}\mathrm{FU} \\
\text { (months) }\end{array}$ & $\begin{array}{l}\text { Outcomes } \\
\text { (assessment) }\end{array}$ & $\begin{array}{c}\text { Data } \\
\text { analysis }\end{array}$ & ЕРНРP \\
\hline Li and Dai (2009) & 76 CBT (38)/I/FTFT & WL (38) & Asia/+/IA & 14 & None & GS (CIAS) & $\mathrm{CO}$ & 3 \\
\hline Li, Garland et al. (2017) & 30 1) MORE (15)/G/FTFT & 2) SUPP (15)/G/FTFT & USA/-/IGD & $\begin{array}{l}\text { 1) } 16 \\
\text { 2) } 16\end{array}$ & 3 & GS (DSM-5 criteria) & ITT & 2 \\
\hline Li, Jin et al. (2017) & 73 1) CBT (36)/G/FTFT & 2) $\mathrm{CBT}+\mathrm{EA}(37) / \mathrm{I}+\mathrm{G} / \mathrm{FTFT}^{\mathrm{f}}$ & Asia/+/IGD & $\begin{array}{l}\text { 1) } 5 \\
\text { 2) } 10\end{array}$ & None & GS (IAT) & $\mathrm{CO}$ & 3 \\
\hline Liu et al. (2013) & 31 1) CBT (16)/G/FTFT & $\begin{array}{l}\text { 2) SM (e.g., written records of } \\
\text { gambling frequency; } \\
\text { determination of target } \\
\text { behaviors) }(15) / \mathrm{G} / \mathrm{SGT}^{\mathrm{f}}\end{array}$ & Asia/-/IA & $\begin{array}{l}\text { 1) } 54 \\
\text { 2) } 24\end{array}$ & None & GS (IAT)FR (h/d) & $\mathrm{CO}$ & 3 \\
\hline Liu et al. (2015) & 46 FT (21)/G/FTFT & WL (25) & Asia/-/IA & 12 & 3 & GS (APIUS)FR (h/w) & $\mathrm{CO}$ & 2 \\
\hline $\begin{array}{l}\text { Pallesen et al. } \\
\text { (2015) }\end{array}$ & $\begin{array}{l}12 \text { IT (CBT; FT; SFT; MI) } \\
\text { (12)/G/FTFT }\end{array}$ & None & $\begin{array}{l}\text { Norway/ } \\
+ \text { /VGA }\end{array}$ & NA & None & GS (GASA; PVP) & $\mathrm{CO}$ & 3 \\
\hline $\begin{array}{l}\text { Park, Kim et al. } \\
\text { (2016) }\end{array}$ & 24 1) $\mathrm{CBT}(12) / \mathrm{G} / \mathrm{FTFT}$ & 2) $\operatorname{VRT}(12) / G / S_{G T}^{f}$ & Asia/-/IGD & $\begin{array}{l}\text { 1) } 16 \\
\text { 2) } 4\end{array}$ & None & GS (YIAS) & $\mathrm{CO}$ & 3 \\
\hline $\begin{array}{l}\text { Pornnoppadol et al. } \\
\text { (2018) }\end{array}$ & $\begin{array}{l}54 \text { 1) IT (CBT }+ \text { skills }+ \\
\text { sports) }(24) / G / F T F T\end{array}$ & 2) $\mathrm{EDU}(30) / \mathrm{G} / \mathrm{FTFT}^{\mathrm{f}}$ & Asia/-/IGD & $\begin{array}{l}\text { NA } \\
\text { 2) } 1\end{array}$ & 6 & GS (GAST) & $\mathrm{CO}$ & 2 \\
\hline $\begin{array}{l}\text { Sakuma et al. } \\
\qquad(2017)^{\mathrm{g}}\end{array}$ & $\begin{array}{l}10 \text { IT (SDiC including CBT; } \\
\text { outdoor cooking; walk } \\
\text { rally; trekking; woodworking) } \\
\text { (10) G/FTFT }\end{array}$ & None & Asia/-/IGD & NA & 3 & $\begin{array}{l}\text { FR (gaming h/d; } \\
\text { h/w; d/w) }\end{array}$ & $\mathrm{CO}$ & 3 \\
\hline Shek et al. (2009) & $\begin{array}{l}22 \text { IT (individual and family } \\
\text { counseling; peer support) } \\
\text { (22)/I/FTFT }\end{array}$ & None & Asia/+/IA & NA & None & GS (CIA-Y; CIA-G) & $\mathrm{CO}$ & 3 \\
\hline Sei et al. (2018) & $46 \mathrm{MI}(\mathrm{PFB})(46) / \mathrm{I} / \mathrm{SGT}$ & None & Asia/+/IA & NA & None & GS (IAT) & $\mathrm{CO}$ & 3 \\
\hline Su et al. (2011) & $\begin{array}{l}59 \mathrm{CBT} \text { (online treatment } \\
\text { program)1) LE } \\
(17) / \mathrm{I} / \mathrm{SGT} 2) \mathrm{NE}(12) / \mathrm{I} / \mathrm{SGT} 3) \\
\mathrm{NI}(14) / \mathrm{I} / \mathrm{SGT}\end{array}$ & NT (16) & Asia/+/IA & $\begin{array}{l}\text { 1) } 0.48 \\
\text { 2) } 0.48 \\
\text { 3) } 0.26\end{array}$ & None & GS (YDQ)FR (h/w) & $\mathrm{CO}$ & 2 \\
\hline $\begin{array}{l}\text { van Rooij et al. } \\
\text { (2012) }\end{array}$ & 7 CBT (7)/I/FTFT & None & $\begin{array}{l}\text { Netherlands/ } \\
\text { +/IA }\end{array}$ & 7.5 & None & GS (CIUS)FR (d/w; h/d) & $\mathrm{CO}$ & 3 \\
\hline $\begin{array}{l}\text { Wartberg et al. } \\
\text { (2014) }\end{array}$ & 18 CBT (18)/G/FTFT & None & Germany/+/IA & 12 & None & $\begin{array}{l}\text { GS (CIUS)FR (h/ } \\
\text { weekdays; } \\
\text { h/weekends) }\end{array}$ & $\mathrm{CO}$ & 3 \\
\hline $\begin{array}{l}\text { Woelfling et al. } \\
\text { (2014) }\end{array}$ & 42 CBT (42)/G+I/FTFT & None & Germany/-/IA & 32 & None & $\begin{array}{l}\text { GS (AICA-S)FR } \\
\text { (h/weekend day) }\end{array}$ & ITT & 3 \\
\hline $\begin{array}{l}\text { Yang and Hao } \\
\text { (2005) }\end{array}$ & $\begin{array}{c}52 \text { IT }(\text { SFBT; FT; CT) } \\
\text { (52)/I/FTFT }\end{array}$ & None & Asia/+/IA & NA & None & GS (YDQ) & $\mathrm{CO}$ & 3 \\
\hline
\end{tabular}


Table 1. Continued

\begin{tabular}{|c|c|c|c|c|c|c|c|c|}
\hline Study/Year & $\begin{array}{l}\text { Treatment group }(N) / \text { Mode of } \\
\text { therapy/Mode of delivery }\end{array}$ & $\begin{array}{l}\text { Control group }(N) / \text { Mode of } \\
\text { therapy/Mode of delivery }{ }^{b}\end{array}$ & $\begin{array}{l}\text { Culture/D/A } \\
(+/-) / \text { IA type }\end{array}$ & $\underset{c^{c}}{\text { Duration } t /}$ & $\begin{array}{c}\mathrm{FU} \\
\text { (months) }\end{array}$ & $\begin{array}{l}\text { Outcomes } \\
\text { (assessment) }\end{array}$ & $\begin{array}{l}\text { Data } \\
\text { analysis }\end{array}$ & ЕРНРP \\
\hline Yang et al. (2017) & $\begin{array}{l}14 \text { 1) } \mathrm{CBT}(14) / \mathrm{G}+\mathrm{I} / \mathrm{FTFT} \\
\text { 2) } \mathrm{EA}(16)^{\mathrm{h}}\end{array}$ & $\mathrm{HC}(16)^{\mathrm{e}}$ & Asia/-/IA & 20 & None & GS (IAT) & $\mathrm{CO}$ & 2 \\
\hline Yao et al. (2017) & $\begin{array}{l}37 \text { IT (RT; MFM) } \\
\text { (18) G/FTFT }\end{array}$ & NT (19) & Asia/+/IGD & 12 & None & GS (CIAS) & $\mathrm{CO}$ & 3 \\
\hline Young (2007) & 114 CBT (114)/I/FTFT & None & USA/+/IA & NA & 6 & $\begin{array}{l}\text { GS (APA; CCU; MSA; } \\
\text { SF)FR (OA) }\end{array}$ & $\mathrm{CO}$ & 3 \\
\hline Young (2013) & $\begin{array}{l}128 \text { CBT modified } \\
(128) / \mathrm{I} / \mathrm{FTFT}\end{array}$ & None & $\mathrm{USA} /+/ \mathrm{IA}$ & NA & 6 & GS (IADQ) & $\mathrm{CO}$ & 3 \\
\hline Zhang (2009) & $\begin{array}{c}70 \text { IT (CBT; sports) } \\
(35) / \mathrm{G} / \mathrm{FTFT}\end{array}$ & NT (35) & Asia/+/IA & 24 & None & GS (IAT) & $\mathrm{CO}$ & 3 \\
\hline Zhang et al. (2009) & 11 CBT (11)/G/FTFT & None & Asia/+/IA & NA & None & GS (IAT) & $\mathrm{CO}$ & 2 \\
\hline Zhang et al. (2016) & $\begin{array}{c}36 \text { IT }(\text { CBI+MFTR }) \\
(20) / G / F T F T\end{array}$ & NT (16) & Asia/+/IGD & 17 & None & GS (CIAS)FR (h/w) & $\mathrm{CO}$ & 2 \\
\hline Zhong et al. (2011) & 57 1) FT (28)/G/FTFT & $\begin{array}{l}\text { 2) IT (military training; } \\
\text { sports; therapy targeting } \\
\text { addictive behaviors) } \\
(29) / \mathrm{G} / \mathrm{FTFT}^{\mathrm{f}}\end{array}$ & Asia/-/IA & $\begin{array}{l}\text { 24.5 } \\
\text { 2) NA }\end{array}$ & 3 & GS (OCS) & $\mathrm{CO}$ & 2 \\
\hline Zhu et al. (2009) & 45 1) CBT (22)/G/FTFT & $\begin{array}{l}\text { 2) } \begin{array}{l}\text { IT }(\mathrm{CBT}+\mathrm{EA}) \\
(23) / \mathrm{I}+\mathrm{G} / \mathrm{FTFT}^{\mathrm{f}}\end{array}\end{array}$ & Asia/+/IA & 52) 10 & None & GS (ISS) & $\mathrm{CO}$ & 2 \\
\hline Zhu et al. (2012) & 73 1) CBT (36)/G/FTFT & $\begin{array}{l}\text { 2) } \text { IT }_{(\mathrm{CBT}+\mathrm{EA})} \\
(37) / \mathrm{I}+\mathrm{G} / \mathrm{FTFT}^{\mathrm{f}}\end{array}$ & Asia/+/IA & 52) 10 & None & GS (IAT) & $\mathrm{CO}$ & 2 \\
\hline \multicolumn{9}{|c|}{ Pharmacological treatments } \\
\hline Bipeta et al. (2015) & $\begin{array}{l}11 \text { Various antidepressants } \\
\text { (after clonazepam was } \\
\text { tapered off in } 3 \text { weeks) } \\
\text { (11)(participants with IA } \\
\text { and OCD) }\end{array}$ & $\begin{array}{l}\text { 2) Various antidepressants } \\
\text { (after clonazepam was } \\
\text { tapered off in } 3 \text { weeks) } \\
\text { (27)(participants with } \\
\text { OCD only) }{ }^{\mathrm{e}}\end{array}$ & India/-/IA & 52 & None & GS (YBOCS; IAT) & NA & 3 \\
\hline $\begin{array}{l}\text { Dell'Osso et al. } \\
\text { (2008) }\end{array}$ & 17 Escitalopram (17) & None & $\mathrm{USA} /+/ \mathrm{IA}$ & 10 & None & $\begin{array}{l}\text { GS (IC-IUD-YBOCS) } \\
\text { FR (h/w) }\end{array}$ & $\mathrm{CO}$ & 3 \\
\hline Han et al. (2009) & 21 Methylphenidate (21)(Concerta) & None & Asia/-/IGD & 8 & None & GS (YIAS-K)FR (h/d) & $\mathrm{CO}$ & 3 \\
\hline Han et al. (2010) & 11 Bupropion SR (11) & None & Asia/-/IGD & 6 & None & GS (YIAS)FR (h/d) & $\mathrm{CO}$ & 3 \\
\hline $\begin{array}{l}\text { Park, Lee et al. } \\
\text { (2016) }\end{array}$ & 86 1) Methylphenidate (44) & $\begin{array}{l}\text { 2) Atomoxetine }(42)^{\mathrm{f}} \\
10-60 \mathrm{mg} / \mathrm{d}\end{array}$ & Asia/-/IGD & 12 & None & GS (YIAS) & $\mathrm{CO}$ & 3 \\
\hline
\end{tabular}


Table 1. Continued

\begin{tabular}{|c|c|c|c|c|c|c|c|c|}
\hline Study/Year & $\begin{array}{l}\text { Treatment group }(N) / \text { Mode of } \\
\text { therapy/Mode of delivery }\end{array}$ & $\begin{array}{l}\text { Control group }(N) / \text { Mode of } \\
\text { therapy/Mode of delivery }\end{array}$ & $\begin{array}{l}\text { Culture/D/A } \\
(+/-) / \text { IA type }\end{array}$ & $\begin{array}{c}\text { Duration } t / \\
c^{c}\end{array}$ & $\begin{array}{c}\mathrm{FU} \\
\text { (months) }\end{array}$ & $\begin{array}{l}\text { Outcomes } \\
\text { (assessment) }\end{array}$ & $\begin{array}{l}\text { Data } \\
\text { analysis }\end{array}$ & ЕРНPP \\
\hline Song et al. (2016) & $\begin{array}{l}119 \text { 1) Bupropion SR (44)2) } \\
\text { Escitalopram (42) }\end{array}$ & NT (33) & Asia/-/IGD & 6 & None & GS (YIAS) & $\mathrm{CO}$ & 2 \\
\hline \multicolumn{9}{|l|}{ Combined treatments } \\
\hline $\begin{array}{l}\text { Han and Renshaw } \\
\text { (2012) }\end{array}$ & $\begin{array}{l}25 \text { 1) Bupropion }+8 \text { sessions } \\
\text { EDU (25) }\end{array}$ & 2) Placebo +8 sessions EDU $(25)^{\mathrm{e}}$ & Asia/+/IGD & 8 & 1 & GS (YIAS)FR (h/w) & $\mathrm{CO}$ & 2 \\
\hline Kim et al. (2012) & $\begin{array}{l}32 \text { 1) Bupropion }+8 \text { sessions } \\
\text { CBT (32) }\end{array}$ & $\begin{array}{l}\text { 2) Bupropion }+10 \text { min. weekly } \\
\text { interviews }(33)^{\mathrm{e}}\end{array}$ & Asia/+/IGD & 8 & 1 & GS (YIAS)FR (h/w) & $\mathrm{CO}$ & 2 \\
\hline Li et al. (2008) & $\begin{array}{l}48 \text { Diverse antidepressants }+ \\
\text { CBT }+ \text { FT }(48)\end{array}$ & None & Asia/+/IA & 4 & None & GS (IRQ) & $\mathrm{CO}$ & 3 \\
\hline Nam et al. (2017) & 30 1) Bupropion+EDU (15) & 2) Escitalopram+EDU $(15)^{\mathrm{f}}$ & Asia/+/IGD & 12 & None & GS (YIAS) & $\mathrm{CO}$ & 2 \\
\hline Santos et al. (2016) & $\begin{array}{l}39 \text { Mixed medications }+10 \\
\text { sessions modified } \\
\text { CBT (39) }\end{array}$ & None & Brazil/+/IA & 10 & None & GS (IAT) & $\mathrm{CO}$ & 3 \\
\hline Yang et al. (2005) & $\begin{array}{l}18 \mathrm{CBT}+\text { parent training }+ \\
\text { Fluoxetine }(18)\end{array}$ & None & Asia/+/IA & 10.5 & None & GS (CIUS) & $\mathrm{CO}$ & 3 \\
\hline
\end{tabular}

Note. A = anxiety; AICA-S = Scale for the Assessment of Internet and Computer Game Addiction; APA = abstinence from problematic sexual online applications; APIUS = Adolescent

Pathological Internet Use Scale; CBI = craving behavior intervention; CBT = Cognitive-behavioral therapy; CCU = control over computer use; CIA-G = Chinese version of the assessment tool based on Goldberg's framework; CIA-Y = Chinese version of Young's Internet Addiction Scale; CIAS = Chinese Internet Addiction Scale; CIAS-R = Chinese Internet Addiction Scale, revised; CIUS = Compulsive Internet Use Scale; CO = completers only; CT = cognitive therapy; $\mathrm{d}=$ day; $\mathrm{D}=$ depression; DQVMIA = Diagnostic Interview for video games, mobile phone, or Internet Addiction (based on the DSM-5 criteria for Internet Gaming Disorder); EA = electroacupuncture; EDU = education program; EPHPP = Effective Public Health Practice Project ( $1=$ strong, $2=$ moderate, 3 = weak rating); F = family; FR = frequency; FT = family therapy; FTFT = face-to-face treatment; FU = follow-up; G = group therapy; GA-MET = group activity-based motivational enhancement therapy; GASA $=$ Game Addiction Scale for Adolescents; GAST $=$ Game Addiction Screening Test; GS $=$ global severity; h = hours; HC $=$ healthy controls; I $=$ individual counseling; IA = internet addiction; IADQ = Internet Addiction Diagnostic Questionnaire; IAT = Internet Addiction Test; IC-IUD-YBOCS = Yale-Browns Obsessive Compulsive Scale adopted for impulsive-compulsive Internet usage disorder; IGD = Internet gaming disorder; IOSRS = Internet Overuse Self-Rating Scale; IRQ = Internet related questionnaire; ISS = Internet addiction selfrating scale; IT $=$ integrative treatment; ITT $=$ intention-to-treat; K-IAS $=$ Korean version of the Internet Addiction Scale; KSAPS $=$ Korean Smartphone Addiction Scale; LE $=$ laboratory environment; MFM = mindfulness meditation; MFTR = mindfulness training; $\mathrm{MI}=$ motivational interviewing; MORE $=$ mindfulness-oriented recovery enhancement; MSA = Mobile Phone Internet Addiction Scale; motivation to stop abusing the Internet; NA = not available; $\mathrm{NE}=$ natural environment; $\mathrm{NI}=$ non-interactive treatment condition; $\mathrm{NT}=$ no treatment; $\mathrm{OA}=$ ability to engage in offline activities; OCD = obsessive-compulsive disorder; OCS = Online Cognition Scale; PI = Positive psychology interventions; PIUQ = Problematic Internet Use Questionnaire; PIUS $=$ Problematic Internet Use Scale; PVP = Problem Video Game Playing Scale; RT = reality therapy; SDiC = Self-discovery camp; SF = ability to abstain from sexually explicit online material; SFBT = solution-focused brief therapy; SFT = solution-focused therapy; SGT = self-guided treatment; SM = self management; SMA = smartphone addiction; S-MAT = Social Media Addiction Test ; SR = sustained release; SUPP = supportive therapy; VGA = videogame addiction; VRT = virtual reality therapy; $\mathrm{w}=$ week; WL = waitlist; YDQ = Young's Diagnostic Questionnaire; YIAS = Young's Internet Addiction Scale; YIAS-K = Young's Internet Addiction Scale, Korean version.

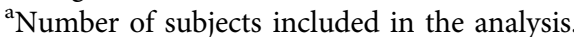

bThe moderators "mode of therapy" and "mode of delivery" were applied for psychological treatments only.

${ }^{c}$ For psychological studies, the duration of treatment was measured using the total number of hours spent in treatment for treatment ( $t$ ) and control groups (c). For pharmacological and combined studies, the duration of treatment was measured using the number of weeks.

${ }^{\mathrm{d}}$ Data for the outcome variable "frequency" were available only for the treatment group.

${ }^{e}$ The control condition was excluded from the analyses due to the incompatibility with the selection criteria.

f The control condition was considered as a separate treatment arm.

${ }^{\mathrm{g}}$ The study reported data from pretreatment to follow-up only.

${ }^{\mathrm{h}}$ The treatment condition was excluded from the analyses due to the incompatibility with the selection criteria. 
Table 2. Characteristics of studies for sex addiction

\begin{tabular}{|c|c|c|c|c|c|c|c|c|}
\hline Study/Year & $\begin{array}{c}\text { Total } \\
N^{\mathrm{a}}\end{array}$ & $\begin{array}{l}\text { Treatment group }(N) / \text { Mode } \\
\text { of therapy/Mode of delivery }\end{array}$ & $\begin{array}{l}\text { Control group }(N) \text { Mode } \\
\text { of therapy/Mode } \\
\text { of delivery }\end{array}$ & $\begin{array}{c}\text { Duration } \\
t / c^{\mathcal{c}} / \mathrm{D} / \mathrm{A}(+/-)\end{array}$ & $\begin{array}{c}\mathrm{FU} \\
\text { (months) }\end{array}$ & Outcomes (assessment) & $\begin{array}{l}\text { Data } \\
\text { analysis }\end{array}$ & ЕРНРP \\
\hline \multicolumn{9}{|c|}{ Psychological treatments } \\
\hline Crosby (2012) & 27 & ACT (14)/I/FTFT & WL (13) & $12 /+$ & $5^{\mathrm{d}}$ & $\begin{array}{l}\text { GS (SCS)FR (pornography } \\
\text { viewing hours/w; modified } \\
\text { version of the DDQ) }\end{array}$ & $\mathrm{CO}$ & 2 \\
\hline $\begin{array}{l}\text { Hallberg et al. } \\
\text { (2017) }\end{array}$ & 10 & CBT (10)/G/FTFT & None & $8 /-$ & 6 & GS (HD:CAS; HDSI) & ITT & 3 \\
\hline $\begin{array}{l}\text { Hallberg et al. } \\
\text { (2019) }\end{array}$ & 137 & CBT (70)/G/FTFT & WL (67) & $8 /-$ & 6 & GS (HD:CAS; SCS) & ITT & 2 \\
\hline $\begin{array}{l}\text { Hardy et al. } \\
\text { (2010) }\end{array}$ & 138 & $\begin{array}{l}\text { CBT (Candeo online program) } \\
\text { (138)/I/SGT }\end{array}$ & None & $26 /+$ & None & $\begin{array}{l}\text { GS (PDR)FR (pornography } \\
\text { use/m; masturbation/m) }\end{array}$ & $\mathrm{CO}$ & 3 \\
\hline $\begin{array}{l}\text { Hart et al. } \\
\quad(2016)\end{array}$ & 49 & MI (49)/G/FTFT & None & $7 /+$ & 3 & GS (SCS) & $\mathrm{CO}$ & 3 \\
\hline $\begin{array}{l}\text { Hartman et al. } \\
(2012) \mathrm{e}^{\mathrm{e}}\end{array}$ & 57 & $\begin{array}{l}\text { IT (program for SA and SA-SUD)/ } \\
\text { I+G/FTFT (57) }\end{array}$ & None & $13 /+$ & 6 & GS (CSBI) & $\mathrm{CO}$ & 3 \\
\hline $\begin{array}{l}\text { Klontz et al. } \\
\text { (2005) }\end{array}$ & 38 & $\begin{array}{l}\text { 1) IT (EXPT; CBT; EDU; M-Medit.), } \\
\text { males (28)/G/FTFT2) IT (EXPT; } \\
\text { CBT; EDU; M-Medit.), females } \\
\text { (10)/G/FTFT }\end{array}$ & None & 1) $1 /+2) 1 /+$ & 6 & GS (GSBI; CGI) & $\mathrm{CO}$ & 3 \\
\hline $\begin{array}{l}\text { Levin et al. } \\
\text { (2017) }\end{array}$ & 11 & ACT $(\mathrm{SHWB})(11) / \mathrm{I} / \mathrm{SGT}$ & None & $8 /+$ & 1.5 & $\begin{array}{l}\text { GS (CPUI)FR (pornography } \\
\text { viewing } h / w \text { ) }\end{array}$ & $\mathrm{CO}$ & 3 \\
\hline $\begin{array}{r}\text { Minarcik } \\
(2016)\end{array}$ & 12 & CBT (12)/I/FTFT & None & $12 /+$ & None & $\begin{array}{l}\text { GS (CLAPS; HBI; SCS)FR } \\
\quad \text { (pornography viewing min./w) }\end{array}$ & $\mathrm{CO}$ & 3 \\
\hline $\begin{array}{l}\text { Orzack et al. } \\
\text { (2006) }\end{array}$ & 35 & IT (RtC; CBT; MI) (35)/G/FTFT & None & $16 /+$ & None & $\begin{array}{l}\text { FR (pornography viewing/w; } \\
\text { OTIS) }\end{array}$ & $\mathrm{CO}$ & 3 \\
\hline $\begin{array}{l}\text { Pachankis et al. } \\
\text { (2015) }\end{array}$ & 63 & $\begin{array}{l}\text { CBT (ESTEEM-SC based on } \\
\text { the UP) }(32) / \mathrm{I} / \mathrm{FTFT}\end{array}$ & WL (31) & $12 /+$ & 3 & GS (SCS) & ITT & 2 \\
\hline $\begin{array}{l}\text { Parsons et al. } \\
\text { (2017) }\end{array}$ & 11 & $\begin{array}{l}\text { CBT (ESTEEM-SC based on } \\
\text { the UP) (11)/I/FTFT }\end{array}$ & None & $12 /+$ & None & GS (SCS) & $\mathrm{CO}$ & 3 \\
\hline $\begin{array}{l}\text { Quadland } \\
\qquad(1985)^{\mathrm{e}}\end{array}$ & 15 & 1) GPT/G/FTFT (15) & $\begin{array}{l}\text { 2) PT for participants } \\
\text { affected by other } \\
\text { problems/I/FTFT } \\
(14)^{\mathrm{f}}\end{array}$ & $20 /+$ & 6 & $\begin{array}{l}\text { FR ( } \mathrm{n} \text { of different sexual } \\
\text { partners/last } 3 \text { months; \% } \\
\text { of sexual partners seen } \\
\text { only once; } \% \text { of sex with } \\
\text { one partner; } \% \text { of sex in } \\
\text { public settings) }\end{array}$ & $\mathrm{CO}$ & 3 \\
\hline $\begin{array}{l}\text { Sadiza et al. } \\
\text { (2011) }\end{array}$ & 10 & CBT (10)/G/FTFT & None & $12 /+$ & None & GS (SCS) & $\mathrm{CO}$ & 3 \\
\hline $\begin{array}{l}\text { Twohig and } \\
\text { Crosby } \\
(2010)\end{array}$ & 6 & ACT (6)/I/FTFT & None & $8 /+$ & 3 & $\begin{array}{l}\text { FR (pornography } \\
\text { viewing } \mathrm{h} / \mathrm{d} \text { ) }\end{array}$ & $\mathrm{CO}$ & 3 \\
\hline Wilson (2010) & 54 & 1) Art therapy (27)/G/FTFT & $\begin{array}{l}\text { 2) modified CBT (TCA) } \\
(27) / \text { FTFT }^{\mathrm{g}}\end{array}$ & 1) $6 /+2) 6 /+$ & 1.5 & GS (HBI-19) & $\mathrm{CO}$ & 2 \\
\hline
\end{tabular}


Table 2. Continued

\begin{tabular}{|c|c|c|c|c|c|c|c|c|}
\hline Study/Year & $\begin{array}{c}\text { Total } \\
N^{\mathrm{a}}\end{array}$ & $\begin{array}{l}\text { Treatment group }(N) / \text { Mode } \\
\text { of therapy/Mode of delivery } \mathrm{b}\end{array}$ & $\begin{array}{c}\text { Control group }(N) \text { Mode } \\
\text { of therapy/Mode } \\
\text { of delivery }{ }^{b}\end{array}$ & $\begin{array}{c}\text { Duration } \\
t / c^{c} / \mathrm{D} / \mathrm{A}(+/-)\end{array}$ & $\begin{array}{c}\mathrm{FU} \\
\text { (months) }\end{array}$ & Outcomes (assessment) & $\begin{array}{c}\text { Data } \\
\text { analysis }\end{array}$ & ЕРНPР \\
\hline \multicolumn{9}{|c|}{ Pharmacological treatments } \\
\hline Kafka (1991) & 10 & $\begin{array}{l}\text { Diverse antidepressants }+ \\
\text { Lithium (10) }\end{array}$ & None & $12 /+$ & None & GS (SOI) & $\mathrm{CO}$ & 3 \\
\hline $\begin{array}{l}\text { Kafka and } \\
\text { Prentky } \\
\text { (1992) }\end{array}$ & 16 & Fluoxetine (16) & None & $12 /+$ & None & GS (SOI) & $\mathrm{CO}$ & 3 \\
\hline Kafka (1994) & $11^{\mathrm{h}}$ & Sertraline (11) & None & $17 /+$ & None & $\begin{array}{l}\text { GS (SOI)FR (fantasizing, urges, } \\
\text { sexual activities min./d) }\end{array}$ & $\mathrm{CO}$ & 3 \\
\hline $\begin{array}{l}\text { Kafka and } \\
\text { Hennen } \\
(2000)\end{array}$ & 26 & $\begin{array}{l}\text { Diverse antidepressants }+ \\
\text { methylphenidate }(26)\end{array}$ & None & $72 /+$ & None & $\begin{array}{l}\text { GS (TSO)FR (fantasizing, urges, } \\
\text { sexual activities min./w) }\end{array}$ & ITT & 3 \\
\hline $\begin{array}{l}\text { Wainberg et al. } \\
\text { (2006) }\end{array}$ & 28 & Citalopram (13) & PLA (15) & $12 /-$ & None & $\begin{array}{l}\text { GS (YBOCS-CSB; CSBI; CGI-CSB) } \\
\text { FR (masturbation, internet use, } \\
\text { pornography use } \mathrm{h} / \mathrm{w} \text { ) }\end{array}$ & ITT & 2 \\
\hline \multicolumn{9}{|c|}{ Combined treatments } \\
\hline $\begin{array}{l}\text { Gola and } \\
\text { Potenza } \\
\text { (2016) }\end{array}$ & 3 & $\mathrm{CBT}+$ Paroxetine $(3)$ & None & $10 /+$ & None & FR (pornography use/w) & $\mathrm{CO}$ & 3 \\
\hline $\begin{array}{l}\text { Scanavino et al. } \\
\text { (2013) }\end{array}$ & 4 & STPGP+various medications (4) & None & $16 /+$ & None & GS (SCS) & $\mathrm{CO}$ & 3 \\
\hline
\end{tabular}

Note. $\mathrm{A}=$ anxiety; ACT $=$ Acceptance and Commitment Therapy; BSI $=$ Brief Symptom Inventory; CBT $=$ cognitive-behavioral therapy; CGI-CSB $=$ Clinical Global Impression Scale adopted for compulsive sexual behavior; CLAPS = Clear Lake Addiction to Pornography Scale; CO = completers only; CPUI = Cyber-Pornography Use Inventory; CSBI = Compulsive Sexual Behavior Inventory; $\mathrm{D}=$ depression; $\mathrm{d}=$ day; DDQ = Daily Drinking Questionnaire; EDU = psychoeducation; EPHPP = Effective Public Health Practice Project $(1=$ strong, $2=$ moderate, $3=$ weak rating); ESTEEM = Effective Skills to Empower Effective Men; EXPT = experiential therapy; FR = frequency; FTFT = face-to-face treatment; FU = follow-up; $\mathrm{G}=$ group setting; GPT = group psychotherapy; GS = global severity; GSBI = Garos Sexual Beavior Inventory; h = hours; HBI = Hypersexual Behavior Inventory; HD:CAS = Hypersexual Disorder:Current Assessment Scale; HDSI = Hypersexual Disorder Screening Inventory; I = individual counseling; IT = integrative treatment; ITT = intention-to-treat; $\mathrm{m}=$ month; M-Medit. = mindfulness meditation; MI = Motivational Interviewing; NA = not available; OTIS = Orzack Time Intensity Survey; PDR = psychological dimensions of recovery (obsessive sexual thoughts, constructive reactions to recovery, positive affect, negative affect, perceptions of agency over the addiction, tendency to deny responsibility for the addiction, meaning in life, connection to others, feelings of being forgiven, awareness of thoughts and tempting situations, healthy pleasure outlets); PLA = placebo; PT = psychotherapy; RtC = Readiness to Change; SA = sexual addiction; SA-SUD = comorbid sexual and substance addiction; SC = sexual compulsivity; SCS = Sexual Compulsivity Scale; SGT = self-guided treatment; SHWB = self-help workbook; SOI = Sexual Outlet Inventory; STPGP = short-term psychodynamic group psychotherapy; TCA = Task Centered Approach; TSO = Total sexual outlet; UP = Unified Protocol for the Transdiagnostic Treatment of Emotional Disorders; W = waitlist; $\mathrm{w}$ = week; YBOCS-CSB = Yale-Brown Obsessive Compulsive Scale modified for compulsive sexual behavior.

${ }^{a}$ Number of subjects included in the analysis.

"The moderators "mode of therapy" and "mode of delivery" were only applied for psychological treatments.

"The duration of treatment was measured by using the number of weeks.

dData from pretreatment to follow-up were available only for the outcome variable "frequency".

e The study reported data from pretreatment to follow-up only.

The control condition was excluded from the analyses due to the incompatibility with the selection criteria.

${ }^{g}$ The control condition was considered as a treatment arm.

${ }^{\mathrm{h}}$ Only participants diagnosed with paraphilia-related disorders were included in the analyses. 
Table 3. Characteristics of studies for compulsive buying

\begin{tabular}{|c|c|c|c|c|c|c|c|c|}
\hline Study/Year & $\begin{array}{c}\text { Total } \\
N^{\mathrm{a}}\end{array}$ & $\begin{array}{l}\text { Treatment group }(N) / \text { Mode of } \\
\text { therapy/Mode of delivery }\end{array}$ & $\begin{array}{l}\text { Control group } \\
(N)\end{array}$ & $\begin{array}{c}\text { Duration } t / c^{c} / D / A \\
(+/-)\end{array}$ & $\begin{array}{c}\mathrm{FU} \\
\text { (months) }\end{array}$ & Outcomes (assessment) & $\begin{array}{l}\text { Data } \\
\text { analysis }\end{array}$ & ЕРНPР \\
\hline \multicolumn{9}{|c|}{ Psychological treatments } \\
\hline Armstrong (2012) & 10 & MBSR (4)/G/FTFT & NT (6) & $8 /+$ & 3 & GS (CBS; YBOCS-SV; IBS) & $\mathrm{CO}$ & 2 \\
\hline Benson et al. (2014) & 11 & $\begin{array}{l}\text { IT (CBT, PSYDYN, PSYEDU, } \\
\text { MI,ACT, mindfulness } \\
\text { elements) (6)/G/FTFT }\end{array}$ & WL (5) & $12 /+$ & 6 & $\begin{array}{l}\text { GS (mod. VCBS; RCBS; } \\
\text { CBS;YBOCS-SV)FR } \\
\text { (min./w spent on buying; } \\
\text { buying episodes/w) }\end{array}$ & $\mathrm{CO}$ & 2 \\
\hline $\begin{array}{l}\text { Filomensky \& Tavares } \\
\text { (2009) }\end{array}$ & 9 & CBT (9)/G/FTFT & None & $20 /+$ & None & GS (YBOCS-SV) & $\mathrm{CO}$ & 3 \\
\hline Mitchell et al. (2006) & 35 & CBT (28)/G/FTFT & WL (7) & $10 /+$ & $6^{\mathrm{e}}$ & $\begin{array}{l}\text { GS (YBOCS-SV; CBS)FR } \\
\text { (buying episodes/w; h } \\
\text { spent buying/w) }\end{array}$ & ITT & 2 \\
\hline Mueller et al. (2008) & 60 & CBT (31)/G/FTFT & WL (29) & $12 /+$ & $6^{\mathrm{e}}$ & $\begin{array}{l}\text { GS (CBS; YBOCS-SV; } \\
\text { G-CBS) }\end{array}$ & ITT & 2 \\
\hline Mueller et al. (2013) & 56 & $\begin{array}{l}\text { 1) CBT (22)/G/FTFT2) } \\
\text { GSH-program (CBT } \\
\text { WB+5 telephone } \\
\text { sessions) }(20) / \mathrm{I} / \mathrm{SGT}\end{array}$ & WL (14) & 1) $10 /+2) 10 /+$ & 6 & GS (CBS; YBOCS-SV) & ITT & 2 \\
\hline \multicolumn{9}{|c|}{ Pharmacological treatments } \\
\hline Black et al. (1997) & 10 & Fluvoxamine (10) & None & $9 /-$ & None & GS (YBOCS-SV) & $\mathrm{CO}$ & 2 \\
\hline Black et al. (2000) & 23 & Fluvoxamine (12) & PLA (11) & $9 /-$ & None & GS (YBOCS-SV) & ITT & 2 \\
\hline Grant et al. (2012) & 9 & Memantine (9) & None & $8 /-$ & None & $\begin{array}{l}\text { GS (YBOCS-SV; mod. } \\
\text { CB-SAS) }\end{array}$ & $\mathrm{CO}$ & 2 \\
\hline Koran et al. (2002) & 24 & Citalopram (24) & None & $12 /+$ & None & GS (YBOCS-SV) & ITT & 2 \\
\hline Koran et al. (2003) & 23 & Citalopram (23) & None & $7 /+$ & None & $\begin{array}{l}\text { GS (YBOCS-SV; } \\
\text { CBS; IBTS) }\end{array}$ & ITT & 2 \\
\hline Koran et al. (2007) & 26 & Escitalopram (26) & None & $7 /+$ & None & GS (YBOCS-SV) & ITT & 3 \\
\hline Ninan et al. (2000) & 37 & Fluvoxamine (20) & PLA (17) & $12 /+$ & None & GS (YBOCS-SV) & ITT & 3 \\
\hline
\end{tabular}

Note. A = anxiety; ACT = Acceptance and Commitment Therapy; CBS = Compulsive Buying Scale; CB-SAS = Compulsive Buying Symptom Assessment Scale (modified version of the Gambling Symptom Assessment Scale; CBT = cognitive-behavioral therapy; CO = completers only; $\mathrm{D}=$ depression; EPHPP $=$ Effective Public Health Practice Project $(1=$ strong, $2=$ moderate, 3 = weak rating); FTFT = face-to-face treatment; FR = frequency; FU = follow-up; G = group setting; G-CBS = Canadian Compulsive Buying Measurement Scale, German version; GS = global severity; GSH = guided self-help; $\mathrm{h}=$ hours; I = individual counseling; IBS = Impulsive Buying Scale; IBTS = Impulse Buying Tendency Scale; ITT = intention to treat analysis; $\mathrm{MBSR}=$ mindfulness-based stress reduction; MI = Motivational Interviewing; NA = not available; NT = no treatment; PLA = placebo control group; PSYDYN = psychodynamic; PSYEDU = psycho-educational; RCBS = Richmond Compulsive Buying Scale; SGT = self-guided treatment; VCBS = Valence Compulsive Buying Scale; WB = workbook; WL = waitlist; $\mathrm{w}=$ week;

YBOCS-SV = Yale-Brown Obsessive Compulsive Scale-Shopping Version.

${ }^{a}$ Number of subjects included in the analysis.

"The moderators "mode of therapy" and "mode of delivery" were only applied for psychological treatments.

${ }^{c}$ The duration of treatment was measured by using the number of weeks.

dData for the outcome variable "frequency" were available only for the treatment group.

${ }^{\mathrm{e}} \mathrm{T}$ The studies were excluded from FU analyses, because only data from posttreatment to FU were reported. 
Table 4. Effect sizes for all types of addictions, outcomes and study designs at posttreatment and at follow-up

\begin{tabular}{|c|c|c|c|c|c|c|c|c|}
\hline Outcome & Type of effect & $k$ & $g$ & $95 \% \mathrm{CI}$ & $z$ & $p$ & $I^{2}$ & FS $N$ \\
\hline \multicolumn{9}{|c|}{ Internet addiction } \\
\hline \multicolumn{9}{|c|}{ Psychological treatments } \\
\hline \multirow[t]{3}{*}{ Global severity } & within-group (post) & 54 & 1.51 & {$[1.29,1.72]$} & 13.79 & $<0.001$ & 93.66 & 18,317 \\
\hline & controlled (post) & 15 & 1.84 & {$[1.37,2.31]$} & 7.268 & $<0.001$ & 83.56 & 1,254 \\
\hline & within-group (FU) & 17 & 1.48 & {$[1.11,1.85]$} & 7.92 & $<0.001$ & 94.61 & 4,221 \\
\hline \multirow[t]{3}{*}{ Frequency } & within-group (post) & 17 & 1.09 & {$[0.73,1.49]$} & 6.02 & $<0.001$ & 92.54 & 1,801 \\
\hline & controlled (post) & 6 & 1.12 & {$[0.41,1.83]$} & 3.08 & $<0.01$ & 78.05 & 69 \\
\hline & within-group (FU) & 6 & 1.06 & {$[0.12,2.00]$} & 2.21 & $<0.05$ & 97.30 & 259 \\
\hline \multicolumn{9}{|c|}{ Pharmacological Treatments } \\
\hline \multirow[t]{3}{*}{ Global severity } & within-group (post) & 8 & 1.13 & {$[0.85,1.42]$} & 7.78 & $<0.001$ & 78.76 & 564 \\
\hline & controlled (post) & 2 & 1.28 & {$[0.85,1.71]$} & 5.85 & $<0.001$ & 0.00 & $-^{a}$ \\
\hline & within-group (FU) & NA & & & & & & \\
\hline \multirow[t]{3}{*}{ Frequency } & within-group (post) & 3 & 0.72 & {$[0.49,0.96]$} & 6.01 & $<0.001$ & 0.00 & 27 \\
\hline & controlled (post) & NA & & & & & & \\
\hline & within-group (FU) & NA & & & & & & \\
\hline \multicolumn{9}{|c|}{ Combined Treatments } \\
\hline \multirow[t]{2}{*}{ Global severity } & within-group (post) & 7 & 2.51 & {$[1.70,3.33]$} & 6.03 & $<0.001$ & 92.99 & 756 \\
\hline & controlled (post) & NA & 215 & & 282 & $<0.01$ & & $\mathrm{a}$ \\
\hline \multirow{3}{*}{ Frequency } & $\begin{array}{l}\text { within-group (FU) } \\
\text { within-group (post) }\end{array}$ & $\begin{array}{l}2 \\
2\end{array}$ & $\begin{array}{l}2.15 \\
2.77\end{array}$ & {$[0.66,3.65]$} & $\begin{array}{c}2.82 \\
11.39\end{array}$ & $<0.001$ & $\begin{array}{l}93.55 \\
14.43\end{array}$ & ${ }^{-}{ }^{a}$ \\
\hline & controlled (post) & NA & & & & & & \\
\hline & within-group (FU) & 2 & 2.69 & {$[2.06,3.32]$} & 8.43 & $<0.001$ & 49.72 & $-{ }^{\mathrm{a}}$ \\
\hline \multicolumn{9}{|c|}{ Sex Addiction } \\
\hline \multicolumn{9}{|c|}{ Psychological Treatments } \\
\hline \multirow[t]{3}{*}{ Global severity } & within-group (post) & 14 & 1.09 & {$[0.74,1.45]$} & 6.03 & $<0.001$ & 92.54 & 1,311 \\
\hline & controlled (post) & 3 & 0.70 & {$[0.42,0.99]$} & 4.87 & $<0.001$ & 7.02 & 19 \\
\hline & within-group (FU) & 10 & 1.00 & {$[0.67,1.32]$} & 6.02 & $<0.001$ & 90.02 & 760 \\
\hline \multirow[t]{3}{*}{ Frequency } & within-group (post) & 6 & 0.75 & {$[0.46,1.03]$} & 5.10 & $<0.001$ & 70.96 & 177 \\
\hline & controlled (post) & 1 & 1.67 & {$[0.82,2.53]$} & 3.83 & $<0.001$ & 0.00 & $-^{\mathrm{a}}$ \\
\hline & within-group (FU) & 4 & 0.83 & {$[0.37,1.29]$} & 3.57 & $<0.001$ & 71.59 & 45 \\
\hline \multicolumn{9}{|c|}{ Pharmacological treatments } \\
\hline \multirow[t]{3}{*}{ Global severity } & within-group (post) & 5 & 1.21 & {$[0.88,1.54]$} & 7.12 & $<0.001$ & 50.42 & 134 \\
\hline & controlled (post) & 1 & 0.14 & {$[-0.58,0.87]$} & 0.38 & 0.70 & 0.00 & $-^{a}$ \\
\hline & within-group (FU) & NA & & & & & & \\
\hline \multirow[t]{3}{*}{ Frequency } & within-group (post) & 3 & 0.87 & {$[0.63,1.12]$} & 6.92 & $<0.001$ & 0.00 & 33 \\
\hline & controlled (post) & 1 & 0.79 & {$[0.04,1.55]$} & 2.06 & $<0.05$ & 0.00 & $-{ }^{a}$ \\
\hline & within-group (FU) & NA & & & & & & \\
\hline \multicolumn{9}{|c|}{ Combined treatments } \\
\hline \multirow[t]{3}{*}{ Global severity } & within-group (post) & 1 & 1.91 & {$[0.75,3.08]$} & 3.22 & $<0.001$ & 0.00 & $-{ }^{\mathrm{a}}$ \\
\hline & controlled (post) & NA & & & & & & \\
\hline & within-group (FU) & NA & & & & & & \\
\hline \multirow[t]{3}{*}{ Frequency } & within-group (post) & 1 & 1.04 & {$[0.22,1.85]$} & 2.49 & $<0.001$ & 0.00 & $-{ }^{\mathrm{a}}$ \\
\hline & controlled (post) & NA & & & & & & \\
\hline & within-group (FU) & NA & & & & & & \\
\hline
\end{tabular}




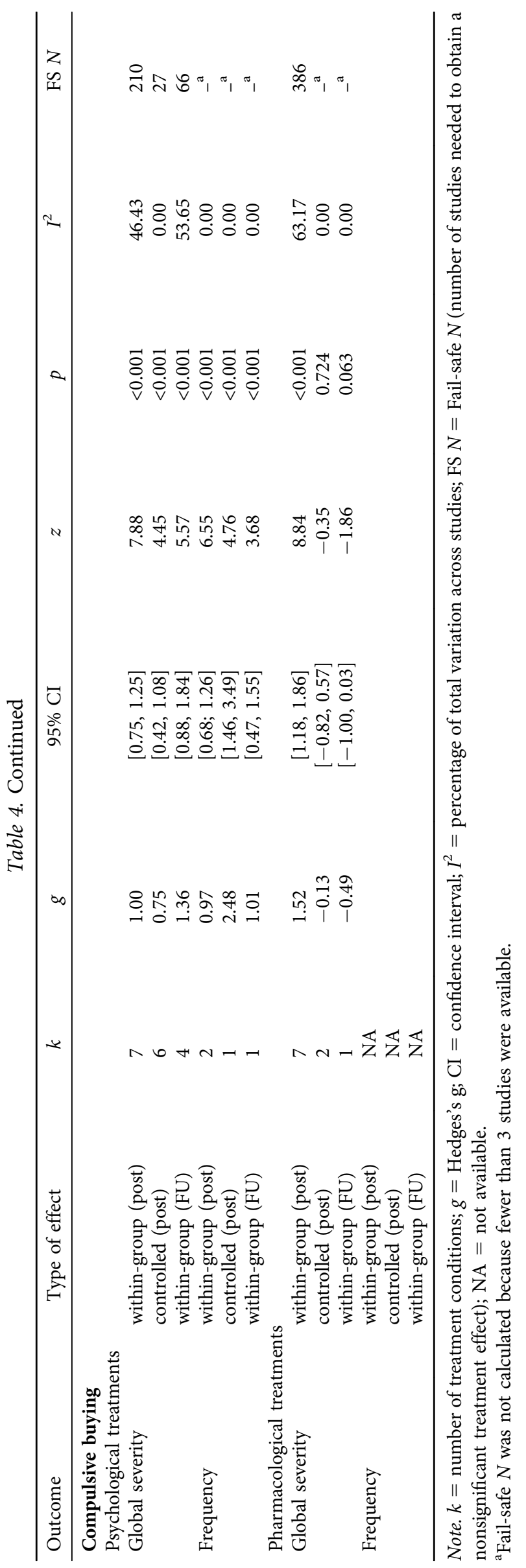

over group settings for the reduction of frequency. Within the $\mathrm{CB}$ category, pharmacological trials using completer analyses produced larger effect sizes than those based on ITT analyses regarding the reduction of global severity.

Moderator analyses on combined treatments were conducted only for the IA category. The results demonstrated that larger effect sizes were associated with CBT-combinations, lower-quality trials, and those examining global IA.

\section{Psychological vs. pharmacological vs. combined treatments}

Regarding IA, combined treatments produced larger effect sizes compared to psychological and pharmacological interventions for the reduction of global severity (psychological vs. combined: $Q_{\text {between }}=7.80, p<0.01$; pharmacological vs. combined: $Q_{\text {between }}=14.69, p<0.001$ ), and frequency (psychological vs. combined: $Q_{\text {between }}=8.73, p<0.01$; pharmacological vs. combined: $Q_{\text {between }}=63.02, p<0.001$ ). Nonsignificant results were found between the effect sizes of pure psychological and pharmacological treatments (global severity: $p=0.173$; frequency: $p=0.492$ ). Considering $C B$, pharmacological treatments showed an advantage over psychological treatments for the reduction of global severity $\left(Q_{\text {between }}=5.45, p<0.05\right)$. No other significant differences between the types of treatments were observed.

\section{Differences between the addiction categories}

Comparisons of the effect sizes across the addiction categories yielded nonsignificant results with respect to psychological interventions (global severity: $p=0.174$; frequency: $p=0.559$ ) and pharmacological interventions (global severity: $p=0.203$; frequency: $p=0.389$ ).

\section{DISCUSSION}

The objective of this paper was to investigate the efficacy of psychological, pharmacological and combined treatments for IA, SA and CB and to identify possible predictors of treatment outcome. Moreover, comparisons between the three types of BAs based on the effect sizes for psychological and pharmacological treatments have been performed for the first time, with the further aim of drawing parallels to disordered gambling and SUDs in terms of treatment response.

We found that psychological treatments effectively reduced the global severity and frequency of IA and SA with the treatment response being maintained over longer periods of time. For $\mathrm{CB}$, psychological treatments were also associated with a large-sized pre-post and pre-follow-up reduction in global severity. Large and moderate short-term gains in terms of both outcome variables were confirmed in controlled study designs, especially regarding IA and in individual studies in the SA and CB categories. These results are in the same range as those obtained in meta-analyses that examined psychological treatments for disordered 
1. Internet addiction

(a) Psychological treatment

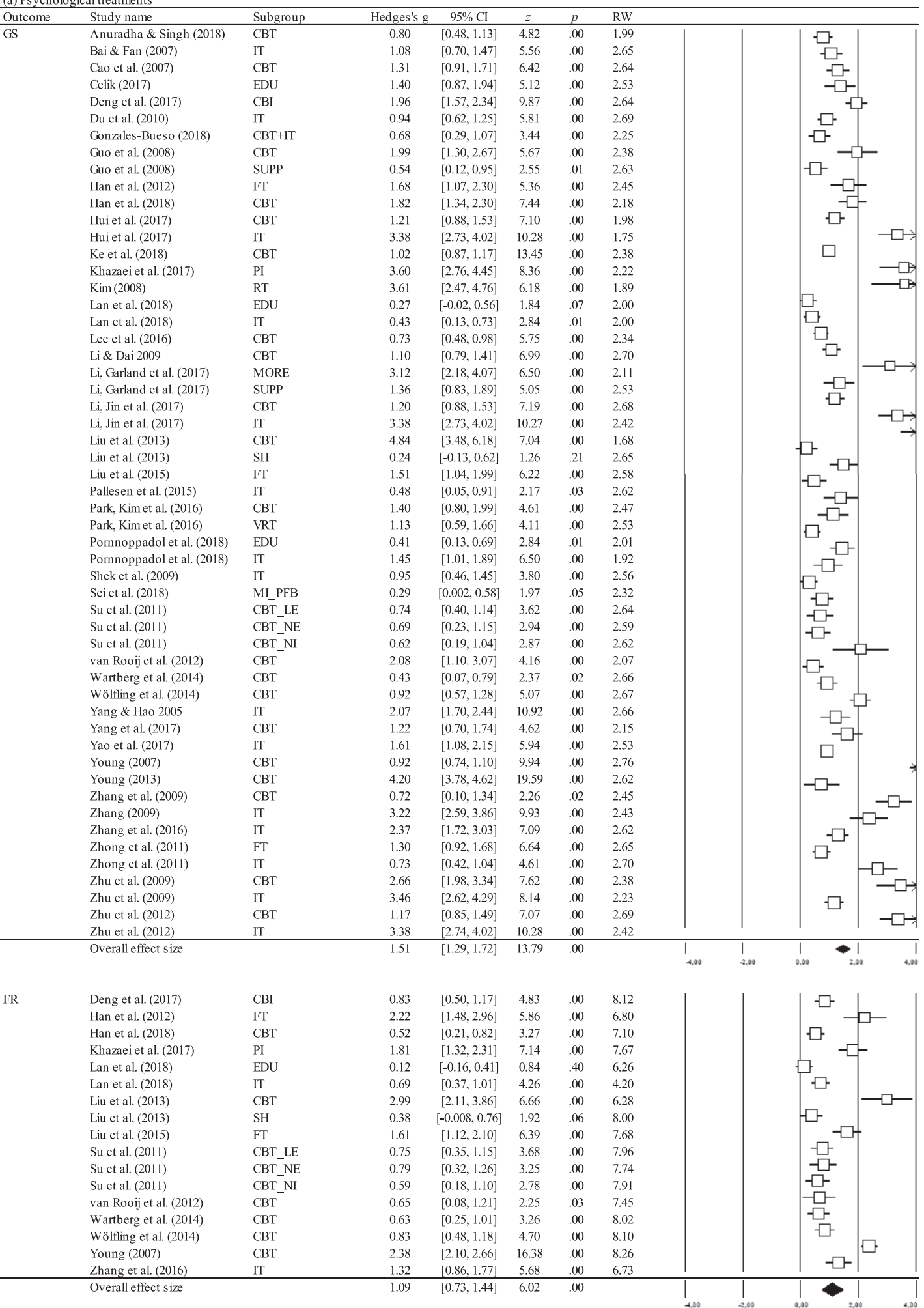

Figure 2. (continued) 


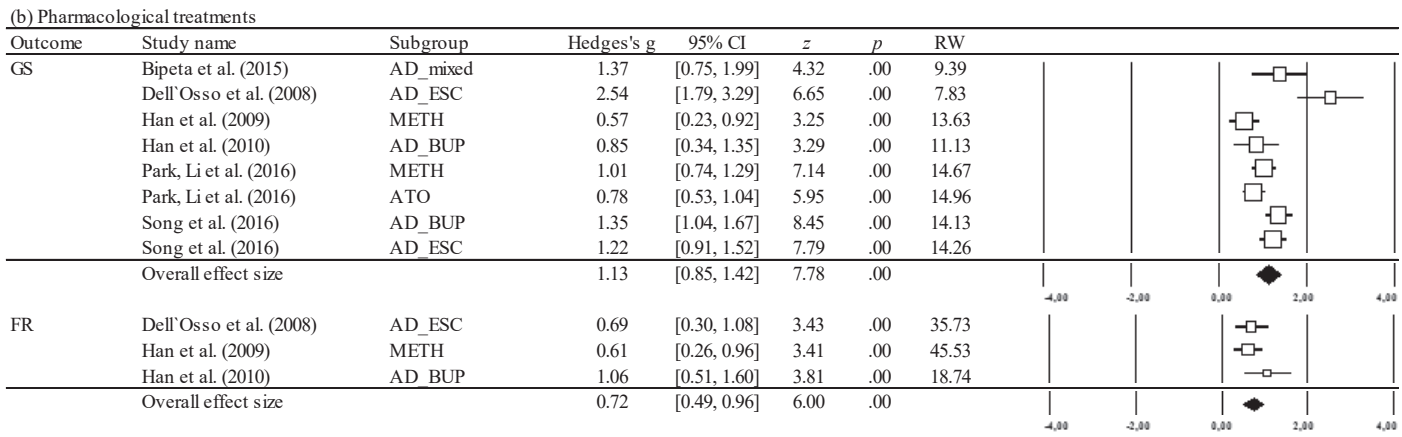

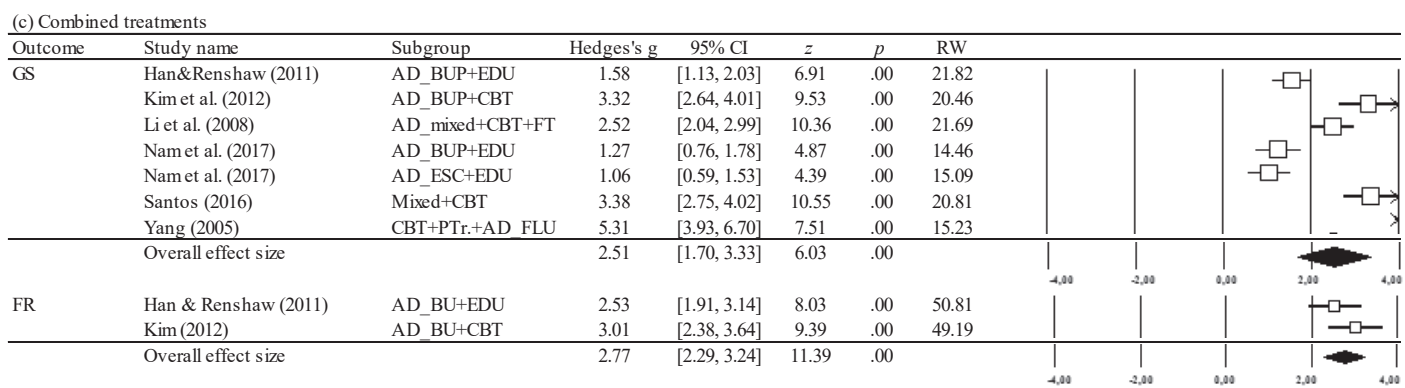

2. Sexaddiction

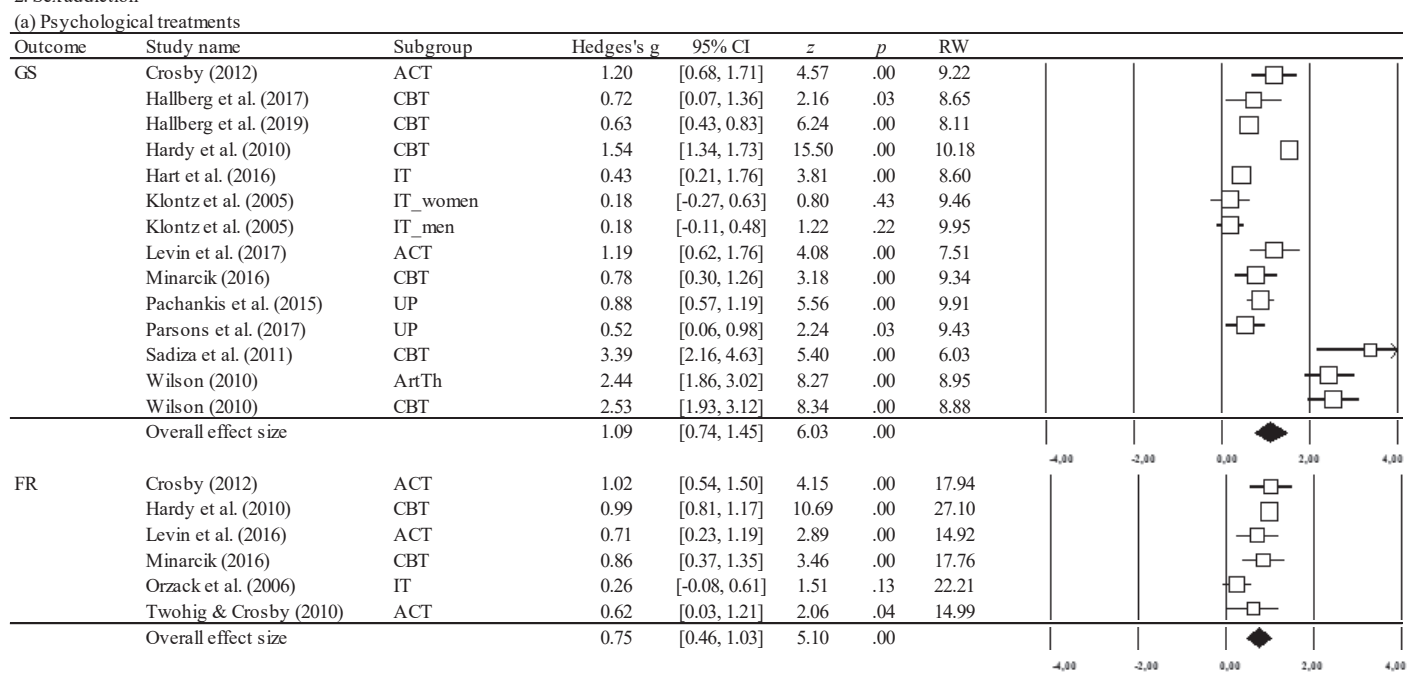

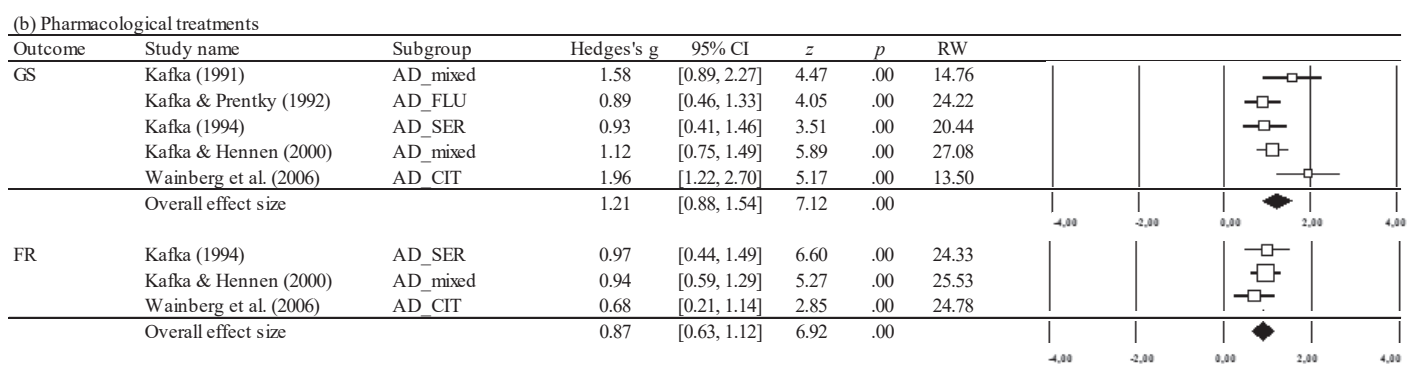

Figure 2. (continued) 


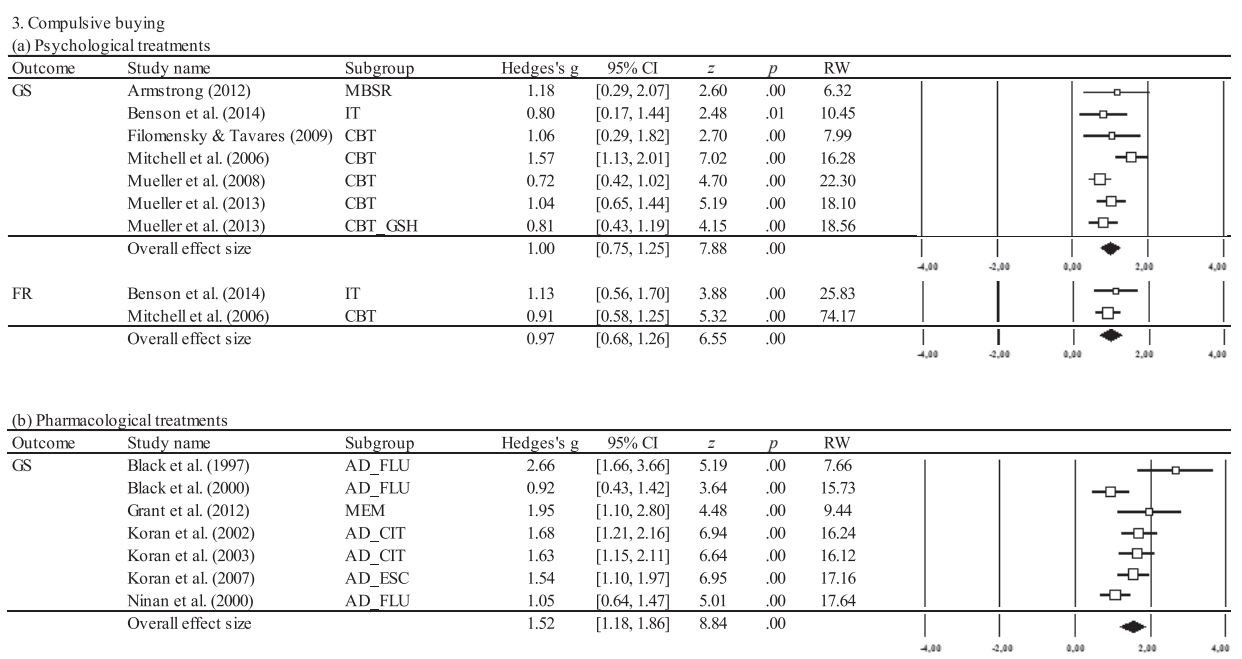

Figure 2. Overall within-group effect sizes for each condition, treatment, and outcome at posttreatment. ACT = acceptance and commitment therapy; $\mathrm{AD}=$ antidepressant; $\mathrm{ArtTh}=$ art therapy; $\mathrm{ATO}=$ atomoxetine; $\mathrm{BUP}=$ bupropion; $\mathrm{CBI}=$ craving behavior intervention; $\mathrm{CBT}=$ cognitive-behavioral therapy; $\mathrm{CIT}=$ citalopram; EDU $=$ education program; $\mathrm{ESC}=$ escitalopram; FLU $=$ fluvoxamine; FT = family therapy; GSH = guided self-help; IT = integrative intervention; LE = laboratory environment; MBRS = mindfulnessbased stress reduction; $\mathrm{MEM}=$ memantine; $\mathrm{METH}=$ methylphenidate; $\mathrm{MI}=$ motivational interviewing; $\mathrm{MORE}=$ mindfulness-oriented recovery enhancement; $\mathrm{NE}=$ natural environment; $\mathrm{NI}=$ non-interactive treatment condition; $\mathrm{PFB}=$ personalized feedback; $\mathrm{PI}=$ positive psychology intervention; PTr = parent training; RT = reality therapy; RW = relative weight; SER = sertraline; SH = self-help; SUPP = supportive therapy; UP = unified protocol for the transdiagnostic treatment of emotional disorders; VRT $=$ virtual reality therapy

gambling (Cowlishaw et al., 2012; Gooding \& Tarrier, 2009; Goslar, Leibetseder, Muench, Hofmann, \& Laireiter, 2017; Leibetseder, Laireiter, Vierhauser, \& Hittenberger, 2011; Pallesen, Mitsem, Kvale, Johnsen, \& Molde, 2005) and SUDs (Dutra et al., 2008; Tripodi, Bender, Litschge, \& Vaughn, 2010).

Although CBT was most commonly used across the three addiction categories, a variety of other psychological approaches proved to be equally effective for reducing problematic behaviors irrespective of the mode of treatment and-particularly with regard to IA-the cultural background. These findings differ from those reported in a recent meta-analysis, which discovered an advantage of CBT over other psychological treatments for the reduction of time spent online, individual counseling, and for studies conducted in the US (Winkler et al., 2013). Discrepancies, however, may be due to the fact that moderator analyses were conducted on pooled within-group and controlled effect sizes and to the addition of the latest research results in our meta-analysis. Among these, the most commonly used approaches included family therapy, which in view of a variety of dysfunctional familial conditions (e.g., Schneider, King, \& Delfabbro, 2017) appear beneficial not only for adolescent problematic internet gamers (e.g., Han, Kim, Lee, \& Renshaw, 2012), but also for adolescents with SUDs (for a review see Filges, Andersen, \& Jørgensen, 2018). Similarly, mindfulness based programs successfully applied for ameliorating symptoms of IA (Li, Garland, et al., 2017) and CB (Armstrong, 2012), and acceptance and commitment therapy implemented for the treatment of SA (e.g., Crosby,
2012) have proven valuable to reduce symptoms of disordered gambling and SUDs (A-tjak et al., 2015; Li, Howard, Garland, McGovern, \& Lazar, 2017; Maynard, Wilson, Labuzienski, \& Whiting, 2018). Integrative programs, which mostly contained CBT elements, produced equally large effect sizes across the three addiction categories, except for the reduction of the frequency of compulsive sexual behaviors. This result, however, was based on a single trial that differed from the others by utilizing the Orzack Time Inventory Survey (OTIS; Orzack, 1999) that appeared "not sufficiently inclusive" (Orzack, Voluse, Wolf, \& Hennen, 2006, p. 354) to measure the frequency of maladaptive computer use. Because Orzack et al. (2006) delivered treatment in group settings, the low effect size of this study also accounted for the disadvantage of group setting compared to individual counseling underlining the importance of using reliable and valid measurement tools (see also Hook, Reid, Penberthy, Davis, \& Jennings, 2014). Furthermore, treatment response appeared to be independent from the type of delivery, with one exception: IA affected individuals receiving FTFTs seemed to profit more from therapy than those included in SGTs. SGTs implemented for the treatment of IA, however, included a considerably lower number of sessions than FTFTs. Therefore, the duration rather than the type of delivery may account for these between-group differences, supporting the results of a recent meta-analysis (Goslar et al., 2017) which indicated that brief SGTs may produce lower levels of improvement than high-intensity, structured selfhelp programs. Evidence for this finding was provided by more intensive SGTs implemented for the treatment of SA 


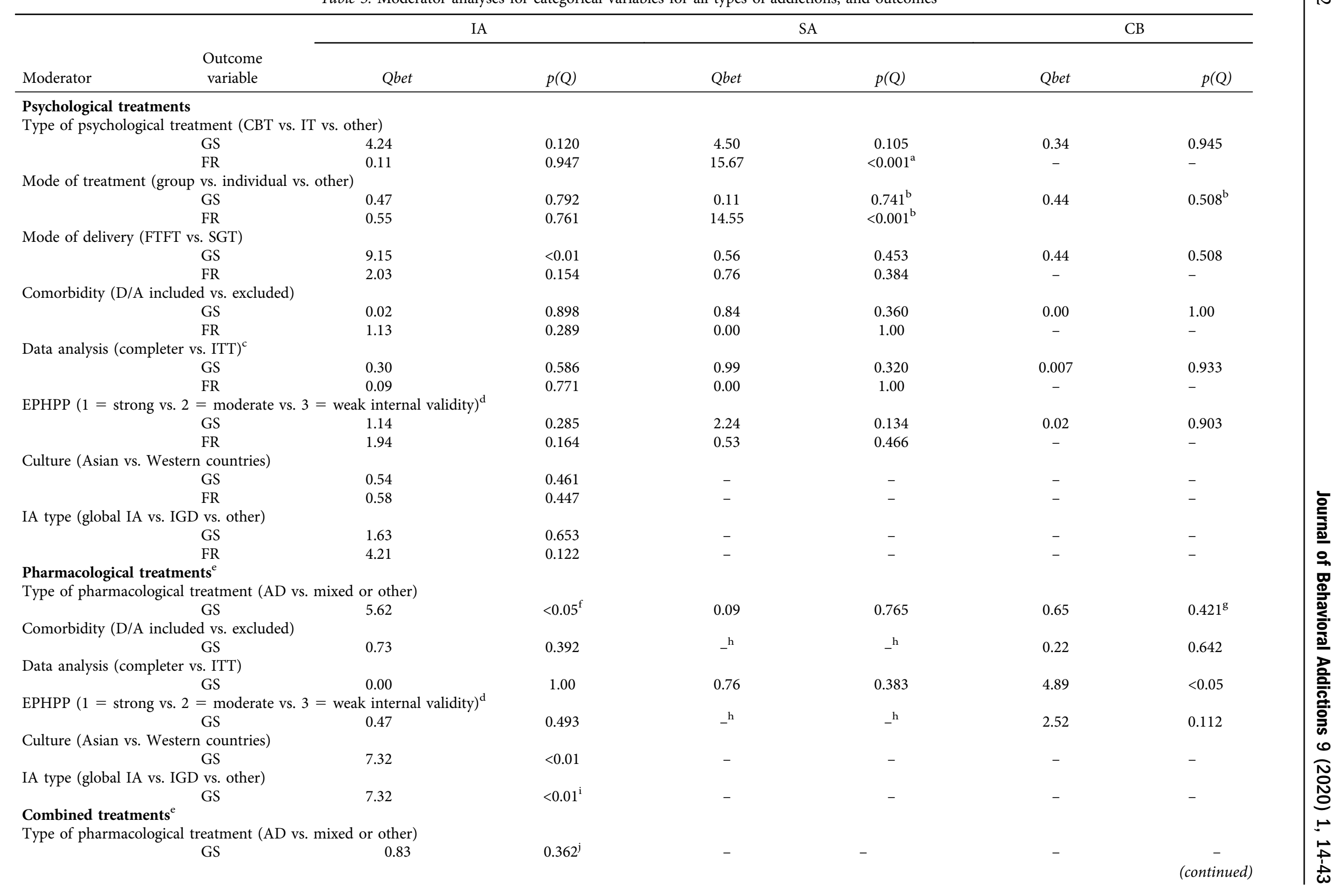


Table 5. Continued

SA

\begin{tabular}{|c|c|c|c|c|c|c|}
\hline \multirow[b]{2}{*}{$\begin{array}{l}\text { Outcome } \\
\text { variable }\end{array}$} & & & \\
\hline & Qbet & $p(Q)$ & Qbet & $p(Q)$ & Qbet & $p(Q)$ \\
\hline \multicolumn{7}{|c|}{ Type of psychological treatment (CBT vs. IT vs. other) } \\
\hline GS & 20.81 & $<0.001^{\mathrm{k}}$ & - & - & - & - \\
\hline \multicolumn{7}{|c|}{ Mode of psychological treatment (group vs. individual vs. other) } \\
\hline GS & 0.29 & $0.592^{\mathrm{b}}$ & - & - & - & - \\
\hline \multicolumn{7}{|l|}{ Comorbidity (D/A included vs. excluded) } \\
\hline GS & 0.00 & 1.00 & - & - & - & - \\
\hline \multicolumn{7}{|l|}{ Data analysis (completer vs. ITT) } \\
\hline GS & 0.00 & 1.00 & - & - & - & - \\
\hline \multicolumn{7}{|c|}{ EPHPP $(1=\text { strong vs. } 2=\text { moderate vs. } 3=\text { weak internal validity })^{\mathrm{d}}$} \\
\hline GS & 6.06 & $<0.05$ & - & - & - & - \\
\hline \multicolumn{7}{|l|}{ Culture (Asian vs. Western countries) } \\
\hline GS & 0.83 & 0.362 & - & - & - & - \\
\hline \multicolumn{7}{|l|}{ IA type (global IA vs. IGD vs. other) } \\
\hline GS & 6.06 & $<0.05^{\mathrm{i}}$ & - & - & - & - \\
\hline
\end{tabular}

Note. $\mathrm{A}=$ anxiety; $\mathrm{AD}=$ antidepressants; $\mathrm{CB}=$ compulsive buying; $\mathrm{CBT}=$ cognitive behavioral therapy; $\mathrm{D}=$ depression; EPHPP = Effective Public Health Practice Project (quality assessment tool for quantitative studies); GS = global severity; FR = frequency; FTFT = face-to-face treatment; IA = internet addiction; IGD = internet gaming disorder; IT = integrative treatment; ITT $=$ intention to treat analysis; Qbet $=$ homogeneity statistic for differences between subgroups; $\mathrm{SA}=$ sex addiction; SGT $=$ self-guided treatment.

${ }^{\mathrm{a}} \mathrm{CBT}: g=0.98 ; 95 \%$ CI $[0.83,1.13] ; p \leq 0.001$; IT: $g=0.25 ; 95 \%$ CI $[-0.08,0.58] ; p=0.132$; Other treatments (i.e., acceptance and commitment therapy): $g=0.80 ; 95 \%$ CI $[0.51,1.10] ; p \leq$ 0.001 .

${ }^{\mathrm{b}}$ Moderator analysis included only two subgroups (group vs. individual)

'Only studies which indicated the type of data analysis were included in the analyses (see Table 1).

${ }^{\mathrm{d}}$ Moderator analysis included only two subgroups $(2=$ moderate; $3=$ weak $)$.

"Moderator analyses on the outcome variable "frequency" were not conducted due to the insufficient number of studies.

${ }^{\mathrm{f}}$ Moderator analysis included only two subgroups (AD vs. other medications [i.e., methylphenidate, atomoxetine]).

${ }^{\mathrm{g}}$ Moderator analysis included only two subgroups (AD vs. other medications [i.e., memantine]).

${ }^{h}$ The results of moderator analyses were not interpreted, because only one study remained in one of the two subgroups.

${ }^{\mathrm{i}}$ Moderator analysis included only two subgroups (IA vs. IGD).

${ }^{\mathrm{j}}$ Moderator analysis included only two subgroups (AD vs. mixed).

${ }^{\mathrm{k}}$ Moderator analysis included only two subgroups (CBT vs. other treatments [i.e., education program]). 
(Hardy, Ruchty, Hull, \& Hyde, 2010; Levin, Heninger, Pierce, \& Twohig, 2017) and CB (Mueller, Arikian, de Zwaan, \& Mitchell, 2013), yielding effect sizes comparable to those found for FTFTs. Accordingly, treatment success increased with the duration of psychotherapy, particularly with regard to the reduction of global severity and frequency of IA. A similar, but nonsignificant result was also observed for the reduction of the global severity of SA. These findings are consistent with those from Asian IA research (Chun et al., 2017), and with those gained from disordered gambling (Goslar et al., 2017; Leibetseder et al., 2011; Pallesen et al., 2005), suggesting that the manifestation of addictive behaviors requires more intensive treatment to achieve improvement.

As with psychological therapies, pharmacological treatments showed large and robust pre-post reductions in pathological symptoms across the three addiction categories. No conclusions, however, can be drawn with respect to the durability of treatment response and the short-term gains of medications over placebo due to the limited amount of data. Moreover, placebo-controlled trials conducted for the treatment of SA and CB were flawed by additional support such as regular therapist contacts including reflection about problematic behaviors (Black, Gabel, Hansen, \& Schlosser, 2000; Wainberg et al., 2006) or concomitant strategies such as keeping shopping diaries (e.g., Black et al., 2000; Ninan et al., 2000) contributing to small between-group differences, and concealing the effect of chemical agents (Black et al., 2000; Ninan et al., 2000; Wainberg et al., 2006). For comparison, short-term gains of pharmacological treatments over placebo for gambling disorder were in the medium range (Goslar, Leibetseder, Muench, Hofmann, \& Laireiter, 2018), similar to those reported for alcohol use disorder, and for a variety of medical diseases and mental health disorders (e.g., Jonas et al., 2014; Leucht, Hierl, Kissling, Dold, \& Davis, 2012).

Moderator analyses showed no significant differences between the classes of medication, although treatment gain for the reduction of global severity for $\mathrm{CB}$ appeared to be overestimated due to larger effect sizes based on completers observed in two trials (Black, Monahan, \& Gabel, 1997; Grant, Odlaug, Mooney, O’Brien, \& Kim, 2012) compared to those obtained from ITT analyses. These trials have also determined the superiority of pharmacological over psychological treatments for the reduction of global severity supporting the use of ITT analysis, which represents a pragmatic statistical approach reflecting more realistic conditions in the context of treatments (e.g., Sedgwick, 2015). Only within the IA category, antidepressants appeared superior to other medications. A closer examination of the data, however, revealed that the subgroup with the higher treatment gain covered adult participants with comorbid depression, and obsessive-compulsive disorders treated with antidepressants, and included the trial with the largest effect size ( $g=2.54$; Dell'Osso et al., 2008). The subgroup with the reduced treatment gain, in turn, included adolescents with comorbid attention deficit hyperactivity disorder (ADHD) treated with psychostimulants (methylphenidate), and contained the trial with the lowest effect size examining individuals with low baseline severity of IA $(g=0.57$; Han et al., 2009). These differences have also affected the moderators "culture" and "IA type". With the two studies removed from moderator analyses, the advantage of antidepressants, and the significant results for the moderators "culture", and "IA type" disappeared. Although treatments in both subgroups yielded beneficial results, differences appeared to be driven by single trials. Therefore, interactions between co-occurring ADHD, medication treatment, age, and culture need investigation if a higher number of studies will be available. Aside from comorbid ADHD, however, disorder-specific improvement was independent from comorbid depression and anxiety, supporting the findings from earlier IA (e.g., Han \& Renshaw, 2012) and disordered gambling research (for a review see Dowling, Merkouris, \& Lorains, 2016).

Across the three addiction categories, mainly serotonin selective reuptake inhibitors (SSRIs) have been examined based on high proportions of comorbid mood disorders (e.g., Kafka, 1991) and-particularly with respect to SA-the inhibitory properties of serotonin on sexual behaviors (e.g., Kafka \& Prentky, 1992). Opioid antagonists (e.g., naltrexone) and glutamatergic medications (e.g., topiramate) were considered only in case studies for treating SA (e.g., Grant \& Kim, 2001; Khazaal \& Zullino, 2006) and CB (e.g., Grant, 2003; Guzman, Filomensky, \& Tavares, 2007) demonstrating beneficial results. Because opioid antagonists and glutamatergic agents proved favorable treatment options for SUDs (Guglielmo et al., 2015; Jonas et al., 2014; Minarini et al., 2017) and disordered gambling (Bartley \& Bloch, 2013; Goslar et al., 2018), these types of drugs seem promising for investigation in larger-scaled and controlled study designs, particularly in the light of high rates of comorbid SUDs observed in BAs (e.g., Grant et al., 2010).

Combined treatments for IA, especially medications in combination with CBT, produced enhanced training effects compared to pure psychological and pharmacological interventions supporting the recommendation of a recent review on IA treatment outcome studies (Przepiorka, Blachnio, Miziak, \& Czuczwar, 2014). The superiority of CBT combinations over those combined with other psychological strategies was assumed to be based on a single trial yielding a very large effect size $(g=5.31$; Yang, Shao, \& Zheng, 2005), affecting also the moderators "quality" and "IA type". With this study removed from subgroup analyses, however, only the advantage of CBT combinations remained significant.

Although most information was provided by IA treatment outcome studies, and data from controlled trials is still limited, psychological and pharmacological approaches showed favorable short-term effects across the three conditions, which are comparable to those applied for substance use and disordered gambling (e.g., Goslar et al., 2017; Grant et al., 2010) supporting our hypotheses. These findings are not sufficient for clarifying the classification of IA, SA and $\mathrm{CB}$ within the spectrum of 
psychiatric disorders due to missing validation of diagnostic criteria, and limited epidemiological, genetic and neurobiological data (e.g., Grant et al., 2010). However, they suggest, that individuals respond equally well to treatments irrespective of the type of addiction. These results fit well into theoretical models of addictive disorders that delineate common underlying mechanisms for both substance-related disorders and BAs (Griffiths, 2005; Jacobs, 1986; Orford, 2001; Shaffer et al., 2004), which can be modified by applying psychological and pharmacological treatments (Potenza et al., 2011). In the light of impaired prefrontal functioning and reward circuits during chronic use of drugs and behaviors (e.g., Nestler, 2005), psychological treatments, particularly CBT-based options, have the potential to alter dysfunctional cognitions and maladaptive behaviors (Kim \& Hodgins, 2018), and to strengthen self-control mechanisms by targeting prefrontal brain areas (Potenza et al., 2011). Pharmacological treatments, in turn, aim at reducing craving and withdrawal symptoms by targeting the reward pathways and neurotransmitter systems (Potenza et al., 2011). Moreover, as observed for the treatment of IA, combinations of CBT and pharmacological treatments may have an additive effect, although the interactions between the two are still unclear (Potenza et al., 2011).

The following limitations should be noted: First, as is true for most meta-analytic reviews, the included studies differed in their methodological quality, although when addressed statistically, we did not observe a systematic bias in the effect sizes due to differences in the quality of the studies. None of the studies, however, achieved the highest rating reflecting limited quality of evidence with respect to selection bias and-due to the preponderance of withingroup study designs-to the identification and control of confounders, and blinding. Therefore, rigorously designed RCTs are necessary, including the monitoring of additional psychosocial support and follow-up data, particularly with respect to pharmacological trials. Moreover, most IA studies included diverse behaviors acted out over the internet (e.g., online gaming, pornography viewing), although research demonstrated differences between the more general concept of IA and specific types of addictive behaviors driven by the internet (Montag et al., 2015). However, we tried to overcome this problem by grouping the studies according to the respective behavior, regardless of the medium used. With regard to co-occurring disorders, we limited moderator analyses to depression and anxiety not only due to their high prevalence among behavioral addictions (e.g., Starcevic \& Khazaal, 2017), but also because these data were most clearly identifiable from the exclusion criteria of the primary studies. Since other conditions often co-occur with BAs (e.g., Grant et al., 2010), and treatment response may be influenced by the nature of comorbidity (Dowling et al., 2016), further studies are encouraged to systematically report the types and rates of co-occurring disorders in order to evaluate this information in future meta-analyses. Most studies also failed to provide information on how the diagnoses had been obtained. The mode of determining the diagnoses, however, may influence their validity (Carlbring et al., 2002; see also Andersson \& Titov, 2014). Future studies should therefore report whether the diagnoses were obtained by clinicians, selfreport, face-to-face, or over the internet. Moreover, future studies are encouraged to directly compare the impact of treatments for individuals with BAs and SUDs in order to investigate similarities and differences between substance-related and non substance-related BAs in terms of treatment response.

Despite these limitations, the results of the present meta-analysis suggest that a variety of psychological interventions are effective for reducing symptoms of IA, especially when delivered face-to-face and conducted over an extended period of time. Although antidepressants and psychostimulants for individuals with cooccurring ADHD improved IA symptoms, CBT combined with antidepressants showed an advantage over monotherapies. Based on the current state of research, CBT and antidepressants appear efficacious for the treatment of SA and CB. Given the demand for treatment, neurobiological research should continue in order to identify parallels between substance-related disorders and possibly addictive behaviors, and to further improve the treatments for these disabling conditions (Grant et al., 2010; Potenza et al., 2011).

Funding sources: This research did not receive direct financial support from any funding agencies in the public, commercial, or not-for-profit sectors.

Author's contribution: Martina Goslar conducted the literature search, extracted the data and performed the analyses. Papers for inclusion in the meta-analysis were screened by Martina Goslar and Max Leibetseder who also validated the data extraction. Anton-Rupert Laireiter supervised these processes. Martina Goslar and Max Leibetseder rated the validity of the studies. Hannah M. Muench supported the organization of the data and gave statistical advice. The manuscript was written by Martina Goslar with comments provided by Hannah M. Muench, Anton-Rupert Laireiter, and Stefan G. Hofmann. All authors contributed to and approved the final manuscript.

Conflict of interest: Martina Goslar declares that she has no conflict of interest. Max Leibetseder declares that he has no conflict of interest. Hannah M. Muench declares that she has no conflict of interest. Dr. Hofmann receives financial support from the Alexander von Humboldt Foundation (as part of the Humboldt prize), NIH/NCCIH (R01AT007257), NIH/NIMH (R01MH099021, U01MH108168), and the James S. McDonnell Foundation $21^{\text {st }}$ Century Science Initiative in Understanding Human Cognition - Special Initiative. He receives compensation for his work as editor from Springer Nature and the Association for Psychological Science, and as an advisor from the Palo Alto Health Sciences and for his work as a Subject Matter Expert from John Wiley \& Sons, Inc. and SilverCloud Health, Inc. He also receives royalties and payments for his editorial work from 
various publishers. Anton-Rupert Laireiter declares that he has no conflict of interest.

Acknowledgments: The authors wish to thank Mrs Xuan Wang und Mrs Yang Zhang who translated the Chinese publications.

\section{APPENDIX}

Formulas for the Effect Size Calculations

To compute the within-group effect sizes, the following formulas were utilized (Borenstein et al, 2005, 2009):

$$
d=\left(\frac{\bar{Y}_{1}-\bar{Y}_{2}}{S_{\text {Difference }}}\right) \sqrt{2(1-r)}
$$

such that $\bar{Y}_{1}$ reflects the pretreatment mean, $\bar{Y}_{2}$ reflects the post-treatment mean, $S_{\text {difference }}$ reflects the standard deviation of the difference, and $r$ reflects the correlation between pretreatment and posttreatment scores. Due to small sample sizes, all effect sizes were corrected for bias using Hedges's $g$ which was computed by multiplying $d$ with the correction factor

$$
J(d f)=1-\frac{3}{4 d f-1}
$$

such that $d f$ represents the degrees of freedom to estimate the within-group standard deviation. These formulas were also applied for the calculation of effect sizes from pretreatment to the latest follow-up. The controlled effect sizes were computed using the following formula:

$$
g=\frac{\left(\bar{\Delta}_{\mathrm{TREAT}}-\bar{\Delta}_{\mathrm{CONT}}\right)}{\sqrt{\frac{\left(n_{\mathrm{TREAT}}-1\right) S D_{\mathrm{TREAT}}^{2}+\left(n_{\mathrm{CONT}}-1\right) S D_{\mathrm{CONT}}^{2}}{n_{\mathrm{Total}}-2}}} \times\left(1-\frac{3}{4\left(n_{\mathrm{Total}}-9\right)}\right),
$$

such that $\bar{\Delta}$ is the mean pre- to post-treatment change, $S D$ is the standard deviation of post-treatment scores, $n$ is the sample size, TREAT refers to the active treatment condition, and CONT refers to the control condition. Following Rosenthal (1991), we estimated the pre-post correlation to be $r=0.70$.

\section{REFERENCES}

A-tjak, J. G., Davis, M. L., Morina, N., Powers, M. B., Smits, J. A., \& Emmelkamp, P. M. (2015). A meta-analysis of the efficacy of acceptance and commitment therapy for clinically relevant mental and physical health problems. Psychotherapy and Psychosomatics, 84, 30-36. https://doi.org/10.1159/000365764.

American Psychiatric Association. (1994). Diagnostic and statistical manual of mental disorders (4th ed.). Washington, DC: Author.

American Psychiatric Association. (2013). Diagnostic and statistical manual of mental disorders (5th ed.). Washington, DC: Author.

Armstrong, A. (2012). Mindfulness and consumerism: A social psychological investigation. (Doctoral dissertation). Retrieved from ProQuest Dissertations and Theses database. (UMI No. U606955).

Andersson, G., \& Titov, N. (2014). Advantages and limitations of internet-based interventions for common mental disorders. World Psychiatry, 13, 4-11. https://doi.org/10.1002/wps.20083.

Bartley, C. A., \& Bloch, M. H. (2013). Meta-analysis: Pharmacological treatment of pathological gambling. Expert Review of Neurotherapeutics, 13, 887-894. https://doi.org/10.1586/ 14737175.2013.814938.

Black, D. W., Gabel, J., Hansen, J., \& Schlosser, S. (2000). A doubleblind comparison of fluvoxamine versus placebo in the treatment of compulsive buying disorder. Annals of Clinical Psychiatry, 12, 205-211. https://doi.org/10.1023/A:1009030425631.

Black, D. W., Monahan, P., \& Gabel, J. (1997). Fluvoxamine in the treatment of compulsive buying. Journal of Clinical Psychiatry, 58, 159-163. https://doi.org/10.4088/JCP.v58n0404.

Borenstein, M., Hedges, L. V., Higgins, J. P. T., \& Rothstein, H. R. (2005). Comprehensive meta-analysis, version 2. Engelwood, NJ: Biostat Inc.

Borenstein, M., Hedges, L. V., Higgins, J. P. T., \& Rothstein, H. R. (2009). Introduction to meta-analysis. Chichester, UK: Wiley.

Brand, M., Snagowski, J., Laier, C., \& Maderwald, S. (2016). Ventral striatum activity when watching preferred pornographic pictures is correlated with symptoms of Internet pornography addiction. NeuroImage, 129, 224-232. https://doi.org/10.1016/j. neuroimage.2016.01.033.

Carlbring, P., Forslin, P., Ljungstrand, P., Willebrand, M., Strandlund, C., Ekselius, L., \& Andersson, G. (2002). Is the internetadministered CIDI-SF equivalent to a clinician-administered SCID interview?. Cognitive Behaviour Therapy, 31, 183-189. https://doi.org/10.1080/165060702321138573.

Chun, J., Shim, H., \& Kim, S. (2017). A meta-analysis of treatment interventions for Internet addiction among Korean adolescents. Cyberpsychology, Behavior and Social Networking, 20, 225-231. https://doi.org/10.1089/cyber.2016.0188.

Cohen, J. (1977). Statistical power analysis for the behavioral sciences. (Rev. ed.). Hillsdale, NJ: Erlbaum.

Cowlishaw, S., Merkouris, S., Dowling, N., Anderson, C., Jackson, A., \& Thomas, S. (2012). Psychological therapies for pathological and problem gambling. Cochrane Database of Systematic Reviews. https://doi.org/10.1002/14651858.CD008937.pub2.

Crosby, J. M. (2012). Acceptance and commitment therapy for the treatment of compulsive pornography use: A randomized clinical trial. (Doctoral dissertation). Retrieved from ProQuest Dissertations and Theses database. (UMI No. 3461332).

Deeks, J. J., Dinnes, J., D’amico, R., Sowden, A., Sakarovitch, C., Song, F., et al. (2003). Evaluating non-randomised intervention studies. Health Technology Assessment, 7, 1-179. https://doi. org/10.3310/hta7270.

Deeks, J. J., Higgins, J. P. T., \& Altman, D. G. (2011). Chapter 9: Analysing data and undertaking meta-analysis. In J. P. T. Higgins, \& S. Green (Eds.), Cochrane handbook of systematic reviews and interventions. Version 5.1.0. (updated March 2011). Available from www.cochrane-handbook.org.

Dell'Osso, B., Hadley, S., Allen, A., Baker, B., Chaplin, W. F., \& Hollander, E. (2008). Escitalopram in the treatment of impulsive-compulsive Internet usage disorder: An open-label trial followed by a double-blind discontinuation phase. Journal of 
Clinical Psychiatry, 69, 452-456. https://doi.org/10.4088/JCP v69n0316.

Derbyshire, K. L., Chamberlain, S. R., Odlaug, B. L., Schreiber, L., \& Grant, J. E. (2014). Neurocognitive functioning in compulsive buying disorder. Annals of Clinical Psychiatry, 26(1), 57-63.

Dowling, N. A., Merkouris, S. S., \& Lorains, F. K. (2016). Interventions for comorbid problem gambling and psychiatric disorders: Advancing a developing field of research. Addictive Behaviors, 58, 21-30. https://doi.org/10.1016/j.addbeh.2016.02. 012.

Dutra, L., Stathopoulou, G., Basden, S. L., Leyro, T. M., Powers, M. B., \& Otto, M. W. (2008). A meta-analytic review of psychosocial interventions for substance use disorders. American Journal of Psychiatry, 165(2), 179-187. https://doi.org/10.1176/ appi.ajp.2007.06111851.

Duval, S., \& Tweedie, R. (2000). Trim and fill: A simple funnelplot-based method of testing and adjusting for publication bias in meta-analysis. Biometrics, 56, 455-463. https://doi.org/10. 1111/j.0006-341X.2000.00455.x.

Egger, M., Smith, G. D., Schneider, M., \& Minder, C. (1997). Bias in meta-analysis detected by a simple, graphical test. BMJ, 315, 629-634. https://doi.org/10.1136/bmj.315.7109.629.

Fauth-Buhler, M., \& Mann, K. (2017). Neurobiological correlates of internet gaming disorder: Similarities to pathological gambling. Addictive Behaviors, 64, 349-356. https://doi.org/10.1016/j. addbeh.2015.11.004.

Filges, T., Andersen, D., \& Jørgensen, A.-M. K. (2018). Effects of Multidimensional Family Therapy (MDFT) on nonopioid drug abuse: A systematic review and meta-analysis. Research on Social Work Practice, 28, 68-83. https://doi.org/10.1177/ 1049731515608241.

Gola, M., Wordecha, M., Sescousse, G., Lew-Starowicz, M., Kossowski, B., Wypych, M., et al. (2017). Can pornography be addictive? An fMRI study of men seeking treatment for problematic pornography use. Neuropsychopharmacology, 42, 20212031. https://doi.org/10.1038/npp.2017.78.

González-Bueso, V., Santamaría, J., Fernández, D., Merino, L., Montero, E., \& Ribas, J. (2018). Association between internet gaming disorder or pathological video-game use and comorbid psychopathology: A comprehensive review. International Journal of Environmental Research and Public Health, 15, 668. https://doi.org/10.3390/ijerph15040668.

Gooding, P., \& Tarrier, N. (2009). A systematic review and metaanalysis of cognitive-behavioural interventions to reduce problem gambling: Hedging our bets?. Behaviour Research and Therapy, 47, 592-607. https://doi.org/10.1016/j.brat.2009.04.002.

Goslar, M., Leibetseder, M., Muench, H. M., Hofmann, S. G., \& Laireiter, A.-R. (2017). Efficacy of face-to-face versus selfguided treatments for disordered gambling: A meta-analysis. Journal of Behavioral Addictions, 6, 142-162. https://doi.org/10. 1556/2006.6.2017.034.

Goslar, M., Leibetseder, M., Muench, H. M., Hofmann, S. G., \& Laireiter, A. R. (2018). Pharmacological treatments for disordered gambling. Journal of Gambling Studies. https://doi.org/10. 1007/s10899-018-09815-y.

Grant, J. E. (2003). Three cases of compulsive buying treated with naltrexone. International Journal of Psychiatry in Clinical Practice, 7(3), 223-225. https://doi.org/10.1080/ 13651500310003219.

Grant, J. E., \& Kim, S. W. (2001). A case of kleptomania and compulsive sexual behavior treated with naltrexone. Annals of Clinical Psychiatry, 13, 229-231. https://doi.org/10.1023/A: 1014626102110.

Grant, J. E., Odlaug, B. L., Mooney, M., O’Brien, R., \& Kim, S. W. (2012). Open-label pilot study of memantine in the treatment of compulsive buying. Annals of Clinical Psychiatry, 24(2), 119-126.

Grant, J. E., Potenza, M. N., Weinstein, A., \& Gorelick, D. A. (2010). Introduction to behavioral addictions. The American Journal of Drug and Alcohol Abuse, 36, 233-241. https://doi. org/10.3109/00952990.2010.491884.

Griffiths, M. (2005). A 'components' model of addiction within a biopsychosocial framework. Journal of Substance Use, 10, 191197. https://doi.org/10.1080/14659890500114359.

Griffiths, M., \& Pontes, H. M. (2014). Internet addiction disorder and internet gaming disorder are not the same. Journal of Addiction Research and Therapy, 5, e124. https://doi.org/10. 4172/2155-6105.1000e124.

Grubbs, J. B., Kraus, S. W., \& Perry, S. L. (2019). Self-reported addiction to pornography in a nationally representative sample: The roles of use habits, religiousness, and moral incongruence. Journal of Behavioral Addictions, 8, 88-93. https://doi.org/10. 1556/2006.7.2018.134.

Guglielmo, R., Martinotti, G., Quatrale, M., Ioime, L., Kadilli, I., Di Nicola, M., \& Janiri, L. (2015). Topiramate in alcohol use disorders: Review and update. CNS Drugs, 29, 383-395. https://doi. org/10.1007/s40263-015-0244-0.

Guzman, C. S., Filomensky, T., \& Tavares, H. (2007). Compulsive buying treatment with topiramate, a case report. Revista Brasileira de Psiquiatria, 29, 383-384. https://doi.org/10.1590/ s1516-44462007000400020.

Hague, B., Hall, J., \& Kellett, S. (2016). Treatments for compulsive buying: A systematic review of the quality, effectiveness and progression of the outcome evidence. Journal of Behavioral Addictions, 5, 379-394. https://doi.org/10.1556/2006.5.2016.064.

Han, D. H., Kim, S. M., Lee, Y. S., \& Renshaw, P. F. (2012). The effect of family therapy on the changes in the severity of on-line game play and brain activity in adolescents with on-line game addiction. Psychiatry Research, 202, 126-131. https://doi.org/10. 1016/j.pscychresns.2012.02.011.

Han, D. H., Lee, Y. S., Na, C., Ahn, J. Y., Chung, U. S., Daniels, M. A., et al. (2009). The effect of methylphenidate on internet video game play in children with attention-deficit/hyperactivity disorder. Comprehensive Psychiatry, 50, 251-256. https://doi. org/10.1016/j.comppsych.2008.08.011.

Han, D. H., \& Renshaw, P. F. (2012). Bupropion in the treatment of problematic online game play in patients with major depressive disorder. Journal of Psychopharmacology, 26, 689-696. https:// doi.org/10.1177/0269881111400647.

Hardy, S. A., Ruchty, J., Hull, T. D., \& Hyde, R. (2010). A preliminary study of an online psychoeducational program for hypersexuality. Sexual Addiction and Compulsivity, 17, 247269. https://doi.org/10.1080/10720162.2010.533999.

Hedges, L. V., \& Olkin, I. (1984). Nonparametric estimators of effect size in meta-analysis. Psychological Bulletin, 96, 573-580. https://doi.org/10.1037/0033-2909.96.3.573. 
Higgins, J. P. T., Thompson, S. G., Deeks, J. J., \& Altman, D. G. (2003). Measuring inconsistency in meta-analyses. BMJ, 327, 557-560. https://doi.org/10.1136/bmj.327.7414.557.

Hoffmann, H., Goodrich, D., Wilson, M., \& Janssen, E. (2014). The role of classical conditioning in sexual compulsivity: A pilot study. Sexual Addiction and Compulsivity, 21, 75-91. https:// doi.org/10.1080/10720162.2014.895460.

Hook, J. N., Reid, R. C., Penberthy, J., Davis, D. E., \& Jennings, D. J. (2014). Methodological review of treatments for nonparaphilic hypersexual behavior. Journal of Sex and Marital Therapy, 40, 294-308. https://doi.org/10.1080/0092623X.2012.751075.

Jacobs, D. F. (1986). A general theory of addictions: A new theoretical model. Journal of Gambling Behavior, 2(1), 15-31.

Jiang, Z., Zhao, X., \& Li, C. (2017). Self-control predicts attentional bias assessed by online shopping-related Stroop in high online shopping addiction tendency college students. Comprehensive Psychiatry, 75, 14-21. https://doi.org/10.1016/ j.comppsych.2017.02.007.

Jonas, D. E., Amick, H. R., Feltner, C., Bobashev, G., Thomas, K., Wines, R., et al. (2014). Pharmacotherapy for adults with alcohol use disorders in outpatient settings: A systematic review and meta-analysis. JAMA, 311(18), 1889-1900. https://doi.org/10.1001/jama.2014.3628.

Kafka, M. P. (1991). Successful antidepressant treatment of nonparaphilic sexual addictions and paraphilias in men. Journal of Clinical Psychiatry, 52(2), 60-65.

Kafka, M. P. (2010). Hypersexual disorder: A proposed diagnosis for DSM-V. Archives of Sexual Behavior, 39, 377-400. https:// doi.org/10.1007/s10508-009-9574-7.

Kafka, M. P., \& Prentky, R. (1992). Fluoxetine treatment of nonparaphilic sexual addictions and paraphilias in men. Journal of Clinical Psychiatry, 53(10), 351-358.

Khazaal, Y., \& Zullino, D. F. (2006). Topiramate in the treatment of compulsive sexual behavior: Case report. BMC Psychiatry, 6, 22. https://doi.org/10.1186/1471-244x-6-22.

Kim, H. S., \& Hodgins, D. C. (2018). Component model of addiction treatment: A pragmatic transdiagnostic treatment model of behavioral and substance addictions. Frontiers in Psychiatry, 9, 1-17. https://doi.org/10.3389/fpsyt.2018.00406.

King, D. L., Delfabbro, P. H., Potenza, M. N., Demetrovics, Z., Billieux, J., \& Brand, M. (2019). Logic, evidence and consensus: Towards a more constructive debate on gaming disorder. Australian and New Zealand Journal of Psychiatry, 53, 10471049. https://doi.org/10.1177/0004867419864435.

King, D. L., Gainsbury, S. M., Delfabbro, P. H., Hing, N., \& Abarbanel, B. (2015). Distinguishing between gaming and gambling activities in addiction research. Journal of Behavioral Addictions, 4, 215-220. https://doi.org/10.1556/ 2006.4.2015.045.

Király, O., Griffiths, M. D., Urbán, R., Farkas, J., Kökönyei, G., Elekes, Z., et al. (2014). Problematic internet use and problematic online gaming are not the same: Findings from a large nationally representative adolescent sample. Cyberpsychology, Behavior, and Social Networking, 17, 749-754. https://doi.org/ 10.1089/cyber.2014.0475.

Kuss, D. J., Pontes, H. M., \& Griffiths, M. D. (2018). Neurobiological correlates in Internet Gaming Disorder: A systematic literature review. Frontiers in Psychiatry, 9, 166. https://doi.org/ 10.3389/fpsyt.2018.00166.
Laier, C., Pawlikowski, M., \& Brand, M. (2014). Sexual picture processing interferes with decision-making under ambiguity. Archives of Sexual Behavior, 43, 473-482. https://doi.org/10. 1007/s10508-013-0119-8.

Laier, C., Schulte, F. P., \& Brand, M. (2013). Pornographic picture processing interferes with working memory performance. Journal of Sex Research, 50, 642-652. https://doi.org/10.1080/ 00224499.2012.716873.

Lawrence, L. M., Ciorciari, J., \& Kyrios, M. (2014). Cognitive processes associated with compulsive buying behaviours and related EEG coherence. Psychiatry Research: Neuroimaging, 221, 97-103. https://doi.org/10.1016/j.pscychresns.2013.10.005.

Leibetseder, M., Laireiter, A.-R., Vierhauser, M., \& Hittenberger, B. (2011). Efficacy and effectiveness of psychological and psychopharmacological treatments in pathological gambling-A metaanalysis. Sucht, 57, 275-285. https://doi.org/10.1024/0939-5911. a000120.

Leucht, S., Hierl, S., Kissling, W., Dold, M., \& Davis, J. M. (2012). Putting the efficacy of psychiatric and general medicine medication into perspective: Review of meta-analyses. British Journal of Psychiatry, 200, 97-106. https://doi.org/10.1192/bjp.bp.111. 096594.

Levin, M. E., Heninger, S. T., Pierce, B. G., \& Twohig, M. P. (2017). Examining the feasibility of acceptance and commitment therapy self-help for problematic pornography viewing: Results from a pilot open trial. The Family Journal, 25, 306-312. https://doi.org/10.1177/1066480717731242.

Li, W., Garland, E. L., McGovern, P., O’Brien, J. E., Tronnier, C., \& Howard, M. O. (2017). Mindfulness-oriented recovery enhancement for internet gaming disorder in U.S. adults: A stage I randomized controlled trial. Psychology of Addictive Behaviors, 31, 393-402. https://doi.org/10.1037/adb0000269.

Li, W., Howard, M. O., Garland, E. L., McGovern, P., \& Lazar, M. (2017). Mindfulness treatment for substance misuse: A systematic review and meta-analysis. Journal of Substance Abuse Treatment, 75, 62-96. https://doi.org/10.1016/j.jsat.2017.01. 008.

Lipsey, M. W., \& Wilson, D. (2000). Practical meta-analysis (applied social research methods). Thousand Oaks, CA: Sage.

Liu, C., Liao, M., \& Smith, D. C. (2012). An empirical review of internet addiction outcome studies in China. Research on Social Work Practice, 22, 282-292. https://doi.org/10.1177/ 1049731511430089.

Maynard, B. R., Wilson, A. N., Labuzienski, E., \& Whiting, S. W. (2018). Mindfulness-based approaches in the treatment of disordered gambling: A systematic review and meta-analysis. Research on Social Work Practice, 28, 348-362. https://doi.org/ 10.1177/1049731515606977.

Mechelmans, D. J., Irvine, M., Banca, P., Porter, L., Mitchell, S., Mole, T. B., et al. (2014). Enhanced attentional bias towards sexually explicit cues in individuals with and without compulsive sexual behaviours. PLoS One, 9, e105476. https://doi.org/ 10.1371/journal.pone.0105476.

Messina, B., Fuentes, D., Tavares, H., Abdo, C. H., \& Scanavino, M. D. T. (2017). Executive functioning of sexually compulsive and non-sexually compulsive men before and after watching an erotic video. The Journal of Sexual Medicine, 14, 347-354. https://doi.org/10.1016/j.jsxm.2016.12.235. 
Minarini, A., Ferrari, S., Galletti, M., Giambalvo, N., Perrone, D., Rioli, G., \& Galeazzi, G. M. (2017). N-acetylcysteine in the treatment of psychiatric disorders: Current status and future prospects. Expert Opinion on Drug Metabolism and Toxicology, 13, 279-292. https://doi.org/10.1080/17425255.2017.1251580.

Moher, D., Liberati, A., Tetzlaff, J., \& Altman, D. G. (2009). Preferred reporting items for systematic reviews and meta-analyses: The PRISMA statement. Annals of Internal Medicine, 151, 264-269. https://doi.org/10.7326/0003-4819-151-4-200908180-00135.

Montag, C., Bey, K., Sha, P., Li, M., Chen, Y. F., Liu, W. Y., et al. (2015). Is it meaningful to distinguish between generalized and specific Internet addiction? Evidence from a cross-cultural study from Germany, Sweden, Taiwan and China. Asia-Pacific Psychiatry, 7, 20-26. https://doi.org/10.1111/appy.12122.

Mueller, A., Arikian, A., de Zwaan, M., \& Mitchell, J. E. (2013). Cognitive-behavioural group therapy versus guided self-help for compulsive buying disorder: A preliminary study. Clinical Psychology and Psychotherapy, 20, 28-35. https://doi.org/10. 1002/cpp.773.

Mueller, A., Brand, M., Claes, L., Demetrovics, Z., de Zwaan, M., Fernández-Aranda, F., et al. (2019). Buying-shopping disorder -Is there enough evidence to support its inclusion in ICD-11? CNS Spectrums, 24, 374-379. https://doi.org/10.1017/ S1092852918001323.

Nestler, E. J. (2005). Is there a common molecular pathway for addiction?. Nature Neuroscience, 8, 1445. https://doi.org/10. 1038/nn1578.

Ninan, P. T., McElroy, S. L., Kane, C. P., Knight, B. T., Casuto, L. S., Rose, S. E., et al. (2000). Placebo-controlled study of fluvoxamine in the treatment of patients with compulsive buying. Journal of Clinical Psychopharmacology, 20, 362-366. https:// doi.org/10.1097/00004714-200006000-00012.

Orford, J. (2001). Addiction as excessive appetite. Addiction, 96, 15-31. https://doi.org/10.1046/j.1360-0443.2001.961152.x.

Orzack, M. H. (1999). How to recognize and treat computer.com addictions Directions in Clinical and Counseloing Psychology. Lesson 2. Vol. 9 (pp. 13-26). New York: The Hatherleigh Co.

Orzack, M. H., Voluse, A. C., Wolf, D., \& Hennen, J. (2006). An ongoing study of group treatment for men involved in problematic Internet-enabled sexual behavior. CyberPsychology and Behavior, 9, 348-360. https://doi.org/10.1089/cpb.2006.9.348.

Pallesen, S., Mitsem, M., Kvale, G., Johnsen, B.-H., \& Molde, H. (2005). Outcome of psychological treatments of pathological gambling: A review and meta-analysis. Addiction, 100, 14121422. https://doi.org/10.1111/j.1360-0443.2005.01204.x.

Pekal, J., Laier, C., Snagowski, J., Stark, R., \& Brand, M. (2018). Tendencies toward Internet-pornography-use disorder: Differences in men and women regarding attentional biases to pornographic stimuli. Journal of Behavioral Addictions, 7, 574583. https://doi.org/10.1556/2006.7.2018.70.

Petry, N. M., Rehbein, F., Ko, C.-H., \& O’Brien, C. P. (2015). Internet gaming disorder in the DSM-5. Current Psychiatry Reports, 17, 72. https://doi.org/10.1007/s11920-015-0610-0.

Pontes, H. M., Kuss, D., \& Griffiths, M. (2015). Clinical psychology of Internet addiction: A review of its conceptualization, prevalence, neuronal processes, and implications for treatment. Neuroscience and Neuroeconomics, 4, 11-23. https://doi.org/10. 2147/NAN.S60982.
Potenza, M. N., Sofuoglu, M., Carroll, K. M., \& Rounsaville, B. J. (2011). Neuroscience of behavioral and pharmacological treatments for addictions. Neuron, 69, 695-712. https://doi.org/ 10.1016/j.neuron.2011.02.009.

Przepiorka, A. M., Blachnio, A., Miziak, B., \& Czuczwar, S. J. (2014). Clinical approaches to treatment of Internet addiction. Pharmacological Reports: PR, 66, 187-191. https://doi.org/10. 1016/j.pharep.2013.10.001.

Raab, G., Elger, C. E., Neuner, M., \& Weber, B. (2011). A neurological study of compulsive buying behaviour. Journal of Consumer Policy, 34, 401. https://doi.org/10.1007/s10603-011-91683.

Rosenthal, R. (1979). The file drawer problem and tolerance for null results. Psychological Bulletin, 86, 638-641. https://doi.org/ 10.1037/0033-2909.86.3.638.

Rosenthal, R. (1991). Meta-analytic procedures for social research. (Vol. 6). Newbury Park, CA: Sage.

Rumpf, H.-J., Achab, S., Billieux, J., Bowden-Jones, H., Carragher, N., Demetrovics, Z., et al. (2018). Including gaming disorder in the ICD-11: The need to do so from a clinical and public health perspective. Journal of Behavioral Addictions, 7, 556-561. https://doi.org/10.1556/2006.7.2018.59.

Saunders, J. B., Hao, W., Long, J., King, D. L., Mann, K., FauthBühler, M., et al. (2017). Gaming disorder: Its delineation as an important condition for diagnosis, management, and prevention. Journal of Behavioral Addictions, 6, 271-279. https://doi. org/10.1556/2006.6.2017.039.

Schmidt, C., Morris, L. S., Kvamme, T. L., Hall, P., Birchard, T., \& Voon, V. (2017). Compulsive sexual behavior: Prefrontal and limbic volume and interactions. Human Brain Mapping, 38, 1182-1190. https://doi.org/10.1002/hbm.23447.

Schneider, L. A., King, D. L., \& Delfabbro, P. H. (2017). Family factors in adolescent problematic Internet gaming: A systematic review. Journal of Behavioral Addictions, 6, 321-333. https:// doi.org/10.1556/2006.6.2017.035.

Sedgwick, P. (2015). Intention to treat analysis versus per protocol analysis of trial data. BMJ, 350, h681. https://doi.org/ 10.1136/bmj.h681.

Seok, J.-W., \& Sohn, J.-H. (2015). Neural substrates of sexual desire in individuals with problematic hypersexual behavior. Frontiers in Behavioral Neuroscience, 9. https://doi.org/10. 3389/fnbeh.2015.00321.

Shaffer, H. J., LaPlante, D. A., LaBrie, R. A., Kidman, R. C., Donato, A. N., \& Stanton, M. V. (2004). Toward a syndrome model of addiction: Multiple expressions, common etiology. Harvard Review of Psychiatry, 12, 367-374. https://doi.org/10.1080/ 10673220490905705.

Snagowski, J., Laier, C., Duka, T., \& Brand, M. (2016). Subjective craving for pornography and associative learning predict tendencies towards cybersex addiction in a sample of regular cybersex users. Sexual Addiction and Compulsivity, 23, 342360. https://doi.org/10.1080/10720162.2016.1151390.

Starcevic, V., \& Khazaal, Y. (2017). Relationships between behavioural addictions and psychiatric disorders: What is known and what is yet to be learned?. Frontiers in Psychiatry, 8. https://doi. org/10.3389/fpsyt.2017.00053.

Starcke, K., Schlereth, B., Domass, D., Schöler, T., \& Brand, M. (2012). Cue reactivity towards shopping cues in female 
participants. Journal of Behavioral Addictions, 2, 17-22. https:// doi.org/10.1556/JBA.1.2012.012.

Sterne, J. A. C., Egger, M., \& Moher, D. (2011). Chapter 10: Addressing reporting biases. In J. P. T. Higgins, \& S. Green (Eds.), Cochrane handbook for systematic reviews of interventions. Version 5.1.0. (updated March 2011). Available from www.cochrane-handbook.org.

Thomas, B. H., Ciliska, D., Dobbins, M., \& Micucci, S. (2004). A process for systematically reviewing the literature: Providing the research evidence for public health nursing interventions. Worldviews on Evidence-Based Nursing, 1, 176-184. https://doi. org/10.1111/j.1524-475X.2004.04006.x.

Thompson, S. G., \& Higgins, J. P. T. (2002). How should metaregression analyses be undertaken and interpreted?. Statistics in Medicine, 21, 1559-1573. https://doi.org/10.1002/sim.1187.

Tripodi, S. J., Bender, K., Litschge, C., \& Vaughn, M. G. (2010). Interventions for reducing adolescent alcohol abuse: A metaanalytic review. Archives of Pediatrics and Adolescent Medicine, 164, 85-91. https://doi.org/10.1001/archpediatrics.2009.235.

Trotzke, P., Starcke, K., Pedersen, A., \& Brand, M. (2014). Cueinduced craving in pathological buying: Empirical evidence and clinical implications. Psychosomatic Medicine, 76, 694-700. https://doi.org/10.1097/PSY.0000000000000126.

Trotzke, P., Starcke, K., Pedersen, A., Müller, A., \& Brand, M. (2015). Impaired decision making under ambiguity but not under risk in individuals with pathological buying-behavioral and psychophysiological evidence. Psychiatry Research, 229, 551-558. https://doi.org/10.1016/j.psychres.2015.05.043.

Voon, V., Mole, T. B., Banca, P., Porter, L., Morris, L., Mitchell, S., et al. (2014). Neural correlates of sexual cue reactivity in individuals with and without compulsive sexual behaviours. PLoS One, 9, e102419. https://doi.org/10.1371/journal.pone.0102419.

Wainberg, M. L., Muench, F., Morgenstern, J., Hollander, E., Irwin, T. W., Parsons, J. T., et al. (2006). A double-blind study of citalopram versus placebo in the treatment of compulsive sexual behaviors in gay and bisexual men. Journal of Clinical Psychiatry, 67, 1968-1973. https://doi.org/10.4088/JCP.v67n1218.

Winkler, A., Doersing, B., Rief, W., Shen, Y., \& Glombiewski, J. A. (2013). Treatment of Internet addiction: A meta-analysis. Clinical Psychology Review, 33, 317-329. https://doi.org/10. 1016/j.cpr.2012.12.005.

World Health Organization (2018). International statistical classification of diseases and related health problems (11th Revision). Retrieved from: https://icd.who.int/browse11/l-m/en.

Yang, R., Shao, Z., \& Zheng, Y. (2005). Comprehensive intervention on Internet addiction of middle school students. Chinese Mental Health Journal, 19(7), 457-459.

\section{REFERENCES INCLUDED IN THE META-ANAL- YSIS}

Anuradha, M., \& Singh, P. (2018). Efficacy of CBT on internet addiction. Journal of Psychosocial Research, 13(1), 109-119.

Armstrong, A. (2012). Mindfulness and consumerism: A social psychological investigation. (Doctoral dissertation). Retrieved from ProQuest Dissertations and Theses database. (UMI No. U606955).

Bai, Y., \& Fan, F.-M. (2007). The effects of group counseling on internet-dependent college students. Chinese Mental Health Journal, 21, 247-250.

Benson, A. L., Eisenach, D., Abrams, L., \& van Stolk-Cooke, K. (2014). Stopping overshopping: A preliminary randomized controlled trial of group therapy for compulsive buying disorder. Journal of Groups in Addiction \& Recovery, 9, 97-125. https://doi.org/10.1080/1556035X.2014.868725.

Bipeta, R., Yerramilli, S. S., Karredla, A. R., \& Gopinath, S. (2015). Diagnostic stability of Internet addiction in obsessive-compulsive disorder: Data from a naturalistic one-year treatment study. Innovations in Clinical Neuroscience, 12(3-4), 14-23. Retrieved from https:/www.ncbi.nlm.nih.gov/pmc/articles/ PMC4420165/pdf/icns_4420112_4420163-4420164_4420114. pdf.

Black, D. W., Gabel, J., Hansen, J., \& Schlosser, S. (2000). A doubleblind comparison of fluvoxamine versus placebo in the treatment of compulsive buying disorder. Annals of Clinical Psychiatry, 12, 205-211. https://doi.org/10.1023/A:1009030425631.

Black, D. W., Monahan, P., \& Gabel, J. (1997). Fluvoxamine in the treatment of compulsive buying. Journal of Clinical Psychiatry, 58, 159-163. https://doi.org/10.4088/JCP.v58n0404.

Cao, F., Su, L.-Y., \& Gao, X.-P. (2007). Control study of group psychotherapy on middle school students with Internet overuse. Chinese Mental Health Journal, 21, 346-349.

Celik, C. B. (2016). Educational intervention for reducing Internet addiction tendencies. Addicta, 3, 375-386. https://doi.org/10. 15805/addicta.2016.3.0021.

Crosby, J. M. (2012). Acceptance and commitment therapy for the treatment of compulsive pornography use: A randomized clinical trial. (Doctoral dissertation). Retrieved from ProQuest Dissertations and These database. (UMI No. 3461332).

Dell'Osso, B., Hadley, S., Allen, A., Baker, B., Chaplin, W. F., \& Hollander, E. (2008). Escitalopram in the treatment of impulsivecompulsive Internet usage disorder: An open-label trial followed by a double-blind discontinuation phase. Journal of Clinical Psychiatry, 69, 452-456. https://doi.org/10.4088/JCP.v69n0316.

Deng, L. Y., Liu, L., Xia, C. C., Lan, J., Zhang, J. T., \& Fang, X. Y. (2017). Craving behavior intervention in ameliorating college students' Internet game disorder: A longitudinal study. Frontiers in Psychology, 8, 526. https://doi.org/10.3389/fpsyg.2017.00526.

Du, Y. S., Jiang, W., \& Vance, A. (2010). Longer term effect of randomized, controlled group cognitive behavioural therapy for Internet addiction in adolescent students in Shanghai. Australian and New Zealand Journal of Psychiatry, 44, 129-134. https://doi.org/10.3109/00048670903282725.

Filomensky, T. Z., \& Tavares, H. (2009). Cognitive restructuring for compulsive buying. Revista Brasileira de Psiquiatria, 31, 77-78. https://doi.org/10.1590/S1516-44462009000100018.

Gola, M., \& Potenza, M. N. (2016). Paroxetine treatment of problematic pornography use: A case series. Journal of Behavioral Addictions, 5, 529-532. https://doi.org/10.1556/2006.5.2016.046.

Gonzalez-Bueso, V., Santamaria, J. J., Fernandez, D., Merino, L., Montero, E., Jimenez-Murcia, S., et al. (2018). Internet gaming disorder in adolescents: Personality, psychopathology and evaluation of a psychological intervention combined with 
parent psychoeducation. Frontiers in Psychology, 9. https://doi. org/10.3389/fpsyg.2018.00787.

Grant, J. E., Odlaug, B. L., Mooney, M., O’Brien, R., \& Kim, S. W. (2012). Open-label pilot study of memantine in the treatment of compulsive buying. Annals of Clinical Psychiatry, 24, 119-126.

Guo, M., Yu, F., \& Chao, X. (2008). Impact evaluation of group counseling on Internet addiction adolescents. Chinese Journal of School Health, 1, 17-19.

Hallberg, J., Kaldo, V., Arver, S., Dhejne, C., \& Oberg, K. G. (2017). A cognitive-behavioral therapy group intervention for hypersexual disorder: A feasibility study. The Journal of Sexual Medicine, 14, 950-958. https://doi.org/10.1016/j.jsxm.2017.05.004.

Hallberg, J., Kaldo, V., Arver, S., Dhejne, C., Jokinen, J., \& Öberg, K. G. (2019). A randomized controlled study of group-administered cognitive behavioral therapy for hypersexual disorder in men. The Journal of Sexual Medicine, 16, 733-745. https://doi. org/10.1016/j.jsxm.2019.03.005.

Han, D. H., Hwang, J. W., \& Renshaw, P. F. (2010). Bupropion sustained release treatment decreases craving for video games and cue-induced brain activity in patients with Internet video game addiction. Experimental and Clinical Psychopharmacology, 18, 297-304. https://doi.org/10.1037/a0020023.

Han, D. H., Kim, S. M., Lee, Y. S., \& Renshaw, P. F. (2012). The effect of family therapy on the changes in the severity of on-line game play and brain activity in adolescents with on-line game addiction. Psychiatry Research, 202, 126-131. https://doi.org/10. 1016/j.pscychresns.2012.02.011.

Han, D. H., Lee, Y. S., Na, C., Ahn, J. Y., Chung, U. S., Daniels, M. A., et al. (2009). The effect of methylphenidate on internet video game play in children with attention-deficit/hyperactivity disorder. Comprehensive Psychiatry, 50, 251-256. https://doi. org/10.1016/j.comppsych.2008.08.011.

Han, D. H., \& Renshaw, P. F. (2012). Bupropion in the treatment of problematic online game play in patients with major depressive disorder. Journal of Psychopharmacology, 26, 689-696. https:// doi.org/10.1177/0269881111400647.

Han, X., Wang, Y., Jiang, W. Q., Bao, X. C., Sun, Y. W., Ding, W. N., et al. (2018). Resting-state activity of prefrontal-striatal circuits in internet gaming disorder: Changes with cognitive behavior therapy and predictors of treatment response. Frontiers in Psychiatry, 9. https://doi.org/10.3389/fpsyt.2018.00341.

Hardy, S. A., Ruchty, J., Hull, T. D., \& Hyde, R. (2010). A preliminary study of an online psychoeducational program for hypersexuality. Sexual Addiction and Compulsivity, 17, 247269. https://doi.org/10.1080/10720162.2010.533999.

Hart, T. A., Stratton, N., Coleman, T. A., Wilson, H. A., Simpson, S. H., Julien, R. E., et al. (2016). A pilot trial of a sexual health counseling intervention for HIV-positive gay and bisexual men who report anal sex without condoms. PLoS One, 11, e0152762. https://doi.org/10.1371/journal.pone.0152762.

Hartman, L. I., Ho, V., Arbour, S., Hambley, J. M., \& Lawson, P. (2012). Sexual addiction and substance addiction: Comparing sexual addiction treatment outcomes among clients with and without comorbid substance use disorders. Sexual Addiction and Compulsivity, 19, 284-309. https://doi.org/10.1080/ 10720162.2012.735515.

Hui, L., Rongjiang, J., Kezhu, Y., Bo, Z., Zhong, Z., Ying, L., et al. (2017). Effect of electro-acupuncture combined with psychological intervention on mental symptoms and P50 of auditory evoked potential in patients with internet addiction disorder. Journal of Traditional Chinese Medicine, 37, 43-48. https://doi.org/10.1016/S0254-6272(17)30025-0.

Kafka, M. P. (1991). Successful antidepressant treatment of nonparaphilic sexual addictions and paraphilias in men. Journal of Clinical Psychiatry, 52, 60-65.

Kafka, M. P. (1994). Sertraline pharmacotherapy for paraphilias and paraphilia-related disorders: An open trial. Annals of Clinical Psychiatry, 6, 189-195.

Kafka, M. P., \& Hennen, J. (2000). Psychostimulant augmentation during treatment with selective serotonin reuptake inhibitors in men with paraphilia-related disorders: A case series. Journal of Clinical Psychiatry, 61, 664-670. https://doi.org/10.4088/JCP. v61n0912.

Kafka, M. P., \& Prentky, R. (1992). Fluoxetine treatment of nonparaphilic sexual addictions and paraphilias in men. Journal of Clinical Psychiatry, 53, 351-358.

Ke, G. N., \& Wong, S. F. (2018). Outcome of the psychological intervention program: Internet use for youth. Journal of Rational-Emotive and Cognitive-Behavior Therapy, 36, 187200. https://doi.org/10.1007/s10942-017-0281-3.

Khazaei, F., Khazaei, O., \& Ghanbari, H. (2017). Positive psychology interventions for Internet addiction treatment. Computers in Human Behavior, 72, 304-311. https://doi.org/10. 1016/j.chb.2017.02.065.

Kim, J.-U. (2008). The effect of a R/T group counseling program on the Internet addiction level and self-esteem of Internet addiction university students. International Journal of Reality Therapy, 27(2), 4-12.

Kim, S. M., Han, D. H., Lee, Y. S., \& Renshaw, P. F. (2012). Combined cognitive behavioral therapy and bupropion for the treatment of problematic on-line game play in adolescents with major depressive disorder. Computers in Human Behavior, 28, 1954-1959. https://doi.org/10.1016/j.chb.2012.05.015.

King, D. L., Kaptsis, D., Delfabbro, P. H., \& Gradisar, M. (2017). Effectiveness of brief abstinence for modifying problematic internet gaming cognitions and behaviors. Journal of Clinical Psychology, 73, 1573-1585. https://doi.org/10.1002/jclp.22460.

Klontz, B. T., Garos, S., \& Klontz, P. T. (2005). The effectiveness of brief multimodal experiential therapy in the treatment of sexual addiction. Sexual Addiction \& Compulsivity, 12, 275-294. https://doi.org/10.1080/10720160500362488.

Koran, L. M., Aboujaoude, E. N., Solvason, B., Gamel, N. N., \& Smith, E. H. (2007). Escitalopram for compulsive buying disorder: A double-blind discontinuation study. Journal of Clinical Psychopharmacology, 27, 225-227. https://doi.org/10.1097/01. jcp.0000264975.79367.f4.

Koran, L. M., Bullock, K. D., Hartston, H. J., Elliott, M. A., \& D'Andrea, V. (2002). Citalopram treatment of compulsive shopping: An open-label study. Journal of Clinical Psychiatry, 63, 704-708. https://doi.org/10.4088/JCP.v63n0808.

Koran, L. M., Chuong, H. W., Bullock, K. D., \& Smith, S. (2003). Citalopram for compulsive shopping disorder: An open-label study followed by double-blind discontinuation. Journal of Clinical Psychiatry, 64, 793-798. https://doi.org/10.4088/JCP.v64n0709.

Lan, Y., Ding, J.-E., Li, W., Li, J., Zhang, Y., Liu, M., \& Fu, H. (2018). A pilot study of a group mindfulness-based cognitive- 
behavioral intervention for smartphone addiction among university students. Journal of Behavioral Addictions, 7, 1171-1176. https://doi.org/10.1556/2006.7.2018.103.

Lee, H., Seo, M. J., \& Choi, T. Y. (2016). The effect of home-based daily journal writing in Korean adolescents with smartphone addiction. Journal of Korean Medical Science, 31, 764-769. https://doi.org/10.3346/jkms.2016.31.5.764.

Levin, M. E., Heninger, S. T., Pierce, B. G., \& Twohig, M. P. (2017). Examining the feasibility of acceptance and commitment therapy self-help for problematic pornography viewing: Results from a pilot open trial. The Family Journal, 25, 306-312. https://doi.org/10.1177/1066480717731242.

Li, G., \& Dai, X.-Y. (2009). Control study of cognitive-behavior therapy in adolescents with Internet addiction disorder. Chinese Mental Health Journal, 23, 457-470.

Li, H., Jin, R. J., Yuan, K. Z., Zheng, B., Zheng, Z., Luo, Y., et al. (2017). Effect of electro-acupuncture combined with psychological intervention on mental symptoms and P50 of auditory evoked potential in patients with internet addiction disorder. Journal of Traditional Chinese Medicine, 37, 43-48. https://doi. org/10.1016/S0254-6272(17)30025-0.

Li, N., Li, G., \& Wang, Y. (2008). The therapeutic effect of 48 internet in patients with addiction. Journal of Psychiatry, 21, 356-359.

Li, W., Garland, E. L., McGovern, P., O’Brien, J. E., Tronnier, C., \& Howard, M. O. (2017). Mindfulness-oriented recovery enhancement for internet gaming disorder in U.S. adults: A stage I randomized controlled trial. Psychology of Addictive Behaviors, 31, 393-402. https://doi.org/10.1037/adb0000269.

Liu, D., Lu, N., He, J.-F., Tang, H., \& Zhou, L.-J. (2013). Effects of internet addiction group counseling on internet using and study management in college students. Chinese Mental Health Journal, 27, 496-501.

Liu, Q. X., Fang, X. Y., Yan, N., Zhou, Z. K., Yuan, X. J., Lan, J., \& Liu, C. Y. (2015). Multi-family group therapy for adolescent Internet addiction: Exploring the underlying mechanisms. Addictive Behaviors, 42, 1-8. https://doi.org/10.1016/j.addbeh.2014.10.021.

Minarcik, J. (2016). Proposed treatment of problematic pornography use: A cognitive-behavioral approach. (Doctoral dissertation). Retrieved from ProQuest Dissertations and These database. (UMI No. 10042888).

Mitchell, J. E., Burgard, M., Faber, R., Crosby, R. D., \& de Zwaan, M. (2006). Cognitive behavioral therapy for compulsive buying disorder. Behaviour Research and Therapy, 44, 1859-1865. https://doi.org/10.1016/j.brat.2005.12.009.

Mueller, A., Arikian, A., de Zwaan, M., \& Mitchell, J. E. (2013). Cognitive-behavioural group therapy versus guided self-help for compulsive buying disorder: A preliminary study. Clinical Psychology and Psychotherapy, 20, 28-35. https://doi.org/10. 1002/cpp.773.

Mueller, A., Mueller, U., Silbermann, A., Reinecker, H., Bleich, S., Mitchell, J. E., \& de Zwaan, M. (2008). A randomized, controlled trial of group cognitive-behavioral therapy for compulsive buying disorder: Posttreatment and 6-month follow-up results. Journal of Clinical Psychiatry, 69, 1131-1138. https://doi.org/10.4088/JCP.v69n0713.

Nam, B., Bae, S., Kim, S. M., Hong, J. S., \& Han, D. H. (2017). Comparing the effects of bupropion and escitalopram on excessive internet game play in patients with major depressive disorder. Clinical Psychopharmacology and Neuroscience, 15, 361-368. https://doi.org/10.9758/cpn.2017.15.4.361.

Ninan, P. T., McElroy, S. L., Kane, C. P., Knight, B. T., Casuto, L. S., Rose, S. E., et al. (2000). Placebo-controlled study of fluvoxamine in the treatment of patients with compulsive buying. Journal of Clinical Psychopharmacology, 20, 362-366. https:// doi.org/10.1097/00004714-200006000-00012.

Orzack, M. H., Voluse, A. C., Wolf, D., \& Hennen, J. (2006). An ongoing study of group treatment for men involved in problematic Internet-enabled sexual behavior. CyberPsychology and Behavior, 9, 348-360. https://doi.org/10.1089/cpb.2006.9.348.

Pachankis, J. E., Hatzenbuehler, M. L., Rendina, H. J., Safren, S. A., \& Parsons, J. T. (2015). LGB-affirmative cognitive-behavioral therapy for young adult gay and bisexual men: A randomized controlled trial of a transdiagnostic minority stress approach. Journal of Consulting and Clinical Psychology, 83, 875-889. https://doi.org/10.1037/ccp0000037.

Pallesen, S., Lorvik, I. M., Bu, E. H., \& Molde, H. (2015). An exploratory study investigating the effects of a treatment manual for video game addiction. Psychological Reports, 117, 490-495. https://doi.org/10.2466/02.PR0.117c14z9.

Park, J. H., Lee, Y. S., Sohn, J. H., \& Han, D. H. (2016). Effectiveness of atomoxetine and methylphenidate for problematic online gaming in adolescents with attention deficit hyperactivity disorder. Human Psychopharmacology: Clinical and Experimental, 31, 427-432. https://doi.org/10.1002/hup.2559.

Park, S. Y., Kim, S. M., Roh, S., Soh, M. A., Lee, S. H., Kim, H., et al. (2016). The effects of a virtual reality treatment program for online gaming addiction. Computer Methods and Programs in Biomedicine, 129, 99-108. https://doi.org/10.1016/j.cmpb.2016.01.015.

Parsons, J. T., Rendina, H., Moody, R. L., Gurung, S., Starks, T. J., \& Pachankis, J. E. (2017). Feasibility of an emotion regulation intervention to improve mental health and reduce HIV transmission risk behaviors for HIV-positive gay and bisexual men with sexual compulsivity. AIDS and Behavior, 21, 1540-1549. https://doi.org/10.1007/s10461-016-1533-4.

Pornnoppadol, C., Ratta-apha, W., Chanpen, S., Wattananond, S., Dumrongrungruang, N., Thongchoi, K., et al. (2018). A comparative study of psychosocial interventions for internet gaming disorder among adolescents aged 13-17 years. International Journal of Mental Health and Addiction, 1-17. https:// doi.org/10.1007/s11469-018-9995-4.

Quadland, M. C. (1985). Compulsive sexual behavior: Definition of a problem and an approach to treatment. Journal of Sex and Marital Therapy, 11, 121-132. https://doi.org/10.1080/ 00926238508406078.

Sadiza, J., Varma, R., Jena, S., \& Singh, T. B. (2011). Group cognitive behaviour therapy in the management of compulsive sex behaviour. International Journal of Criminal Justice Sciences, 6, 309-325.

Sakuma, H., Mihara, S., Nakayama, H., Miura, K., Kitayuguchi, T., Maezono, M., et al. (2017). Treatment with the Self-Discovery Camp (SDiC) improves Internet gaming disorder. Addictive Behaviors, 64, 357-362. https://doi.org/10.1016/j.addbeh.2016.06.013.

Santos, V. A., Freire, R., Zugliani, M., Cirillo, P., Santos, H. H., Nardi, A. E., \& King, A. L. (2016). Treatment of Internet addiction with anxiety disorders: Treatment protocol and 
preliminary before-after results involving pharmacotherapy and modified cognitive behavioral therapy. Jmir Research Protocols, 5, e46. https://doi.org/10.2196/resprot.5278.

Scanavino, M. D., Kimura, C. M. S., Messina, B., Abdo, C. H. N., \& Tavares, H. (2013). Five cases of sexual addiction under shortterm psychodynamic group psychotherapy. Revista De Psiquiatria Clinica, 40, 208-209. https://doi.org/10.1590/S010160832013000500007.

Sei, J., Minai, J., Funato, K., Hara, H., \& Ayukawa, M. (2018). Effect of web-based intervention on internet addiction among adolescents: A quasi-experimental trial. Journal of Adolescent Health, 62, S126-S126. https://doi.org/10.1016/j. jadohealth.2017.11.256.

Shek, D. T. L., Tang, V. M. Y., \& Lo, C. Y. (2009). Evaluation of an Internet addiction treatment program for Chinese adolescents in Hong Kong. Adolescence, 44, 359-373.

Song, J., Park, J. H., Han, D. H., Roh, S., Son, J. H., Choi, T. Y., et al. (2016). Comparative study of the effects of bupropion and escitalopram on internet gaming disorder. Psychiatry and Clinical Neurosciences, 70, 527-535. https://doi.org/10. $1111 / \mathrm{pcn} .12429$.

Su, W., Fang, X., Miller, J. K., \& Wang, Y. (2011). Internet-based intervention for the treatment of online addiction for college students in China: A pilot study of the Healthy Online Self-Helping Center. Cyberpsychology, Behavior, and Social Networking, 14, 497503. https://doi.org/10.1089/cyber.2010.0167.

Twohig, M. P., \& Crosby, J. M. (2010). Acceptance and commitment therapy as a treatment for problematic Internet pornography viewing. Behavior Therapy, 41, 285-295. https://doi.org/ 10.1016/j.beth.2009.06.002.

Van Rooij, A. J., Zinn, M. F., Schoenmakers, T. M., \& van de Mheen, D. (2012). Treating internet addiction with cognitivebehavioral therapy: A thematic analysis of the experiences of therapists. International Journal of Mental Health and Addiction, 10, 69-82. https://doi.org/10.1007/s11469-0109295-0.

Wainberg, M. L., Muench, F., Morgenstern, J., Hollander, E., Irwin, T. W., Parsons, J. T., O'Leary, A. (2006). A double-blind study of citalopram versus placebo in the treatment of compulsive sexual behaviors in gay and bisexual men. Journal of Clinical Psychiatry, 67, 1968-1973. https://doi.org/10.4088/JCP.v67n1218.

Wartberg, L., Thomsen, M., Moll, B., \& Thomasius, R. (2014). Pilot study on the effectiveness of a cognitive behavioural group programme for adolescents with pathological internet use. Praxis der Kinderpsychologie und Kinderpsychiatrie, 63(1), 21-35.

Wilson, M. D. (2010). A comparative study of art therapy and cognitive behavioral therapy in the treatment of sexually addictive behaviors and an investigation into the relationship between shame and sexually addicted behaviors in adults. (Doctoral dissertation). Retrieved from ProQuest Dissertations and These database. (UMI No. 3397362).

Woelfling, K., Beutel, M. E., Dreier, M., \& Muller, K. W. (2014). Treatment outcomes in patients with internet addiction: A clinical pilot study on the effects of a cognitive-behavioral therapy program. BioMed Research International, 2014, 1-8. https://doi.org/10.1155/2014/425924.

Yang, F., \& Hao, W. (2005). The effect of integrated psychosocial intervention on 52 adolescents with Internet addiction disorder. Chinese Journal of Clinical Psychology, 13, 343-345.

Yang, R., Shao, Z., \& Zheng, Y. (2005). Comprehensive intervention on Internet addiction of middle school students. Chinese Mental Health Journal, 19, 457-459.

Yao, Y.-W., Chen, P.-R., Li, C.-S. R., Hare, T. A., Li, S., Zhang, J.-T., et al. (2017). Combined reality therapy and mindfulness meditation decrease intertemporal decisional impulsivity in young adults with Internet gaming disorder. Computers in Human Behavior, 68, 210-216. https://doi.org/10.1016/j.chb. 2016.11.038.

Yang, Y., Li, H., Chen, X. X., Zhang, L. M., Huang, B. J., \& Zhu, T. M. (2017). Electro-acupuncture treatment for internet addiction: Evidence of normalization of impulse control disorder in adolescents. Chinese Journal of Integrative Medicine, 23, 837844. https://doi.org/10.1007/s11655-017-2765-5.

Young, K. S. (2007). Cognitive behavior therapy with Internet addicts: Treatment outcomes and implications. CyberPsychology and Behavior, 10, 671-679. https://doi.org/10.1089/cpb.2007. 9971.

Young, K. S. (2013). Treatment outcomes using CBT-IA with Internet-addicted patients. Journal of Behavioral Addictions, 2, 209-215. https://doi.org/10.1556/JBA.2.2013.4.3.

Zhang, J. T., Ma, S. S., Li, C. S. R., Liu, L., Xia, C. C., Lan, J., et al. (2016). Craving behavioral intervention for internet gaming disorder: Remediation of functional connectivity of the ventral striatum. Addiction Biology, 23, 337-346. https://doi.org/10. $1111 /$ adb.12474.

Zhang, L. (2009). The applications of group mental therapy and sports exercise prescriptions in the intervention of Internet addiction disorder. Psychological Science (China), 32, 738-741.

Zhang, R.-H., Chen, W.-P., \& Dong, X.-L. (2009). Effect of cognitive-behavioral group therapy on Internet addiction disorders among senior middle school students in small sample. Chinese Journal of School Health, 30, 1104-1106.

Zhong, X., Zu, S., Sha, S., Tao, R., Zhao, C., Yang, F., et al. (2011). The effect of a family-based intervention model on Internetaddicted Chinese adolescents. Social Behavior and Personality, 39, 1021-1034. https://doi.org/10.2224/sbp.2011.39.8.1021.

Zhu, T., Jin, R., \& Zhong, X. (2009). Clinical effect of electroacupuncture combined with psychologic interference on patient with Internet addiction disorder. Chinese Journal of Integrated Traditional and Western Medicine, 29, 212-214.

Zhu, T. M., Li, H., Jin, R. J., Zheng, Z., Luo, Y., Ye, H., \& Zhu, H. M. (2012). Effects of electroacupuncture combined psycho-intervention on cognitive function and event-related potentials P300 and mismatch negativity in patients with internet addiction. Chinese Journal of Integrative Medicine, 18, 146-151. https:// doi.org/10.1007/s11655-012-0990-5.

This is an open-access article distributed under the terms of the Creative Commons Attribution-NonCommercial 4.0 International License (https://creativecommons.org/ licenses/by-nc/4.0/), which permits unrestricted use, distribution, and reproduction in any medium for non-commercial purposes, provided the original author and source are credited, a link to the CC License is provided, and changes - if any - are indicated. 